JOURNAL OF

SYNCHROTRON

RADIATION
Received 8 January 2016

Accepted 9 May 2016

Edited by A. Momose, Tohoku University, Japan

Keywords: coherent X-ray diffraction imaging; $X$-ray free-electron laser; frozen-hydrated non-crystalline specimens; cryogenic X-ray diffraction experiment; structures of cells and cellular organelles

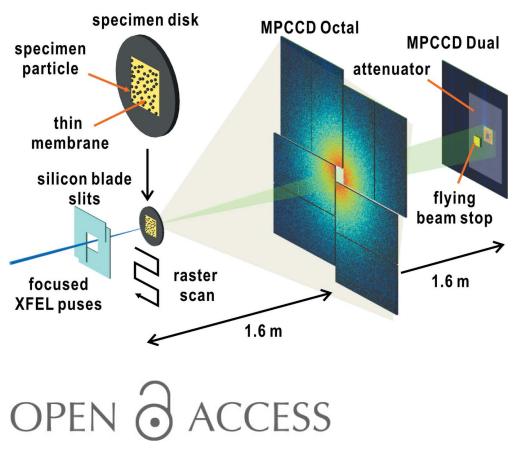

\section{Specimen preparation for cryogenic coherent $X$-ray diffraction imaging of biological cells and cellular organelles by using the X-ray free-electron laser at SACLA}

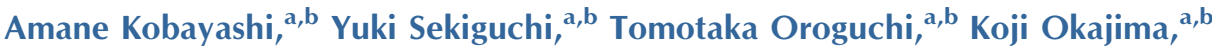 \\ Asahi Fukuda, ${ }^{a, b}$ Mao Oide, ${ }^{a, b}$ Masaki Yamamoto ${ }^{b}$ and Masayoshi Nakasako ${ }^{a}, b_{*}$ \\ aDepartment of Physics, Faculty of Science and Technology, Keio University, 3-14-1 Hiyoshi, Kohoku-ku, \\ Yokohama, Kanagawa 223-8522, Japan, and ${ }^{\mathbf{b}}$ RIKEN SPring-8 Center, 1-1-1 Kouto, Sayo, Hyogo 679-5148, Japan. \\ *Correspondence e-mail: nakasako@phys.keio.ac.jp
}

Coherent X-ray diffraction imaging (CXDI) allows internal structures of biological cells and cellular organelles to be analyzed. CXDI experiments have been conducted at $66 \mathrm{~K}$ for frozen-hydrated biological specimens at the SPring-8 Angstrom Compact Free-Electron Laser facility (SACLA). In these cryogenic CXDI experiments using X-ray free-electron laser (XFEL) pulses, specimen particles dispersed on thin membranes of specimen disks are transferred into the vacuum chamber of a diffraction apparatus. Because focused single XFEL pulses destroy specimen particles at the atomic level, diffraction patterns are collected through raster scanning the specimen disks to provide fresh specimen particles in the irradiation area. The efficiency of diffraction data collection in cryogenic experiments depends on the quality of the prepared specimens. Here, detailed procedures for preparing frozenhydrated biological specimens, particularly thin membranes and devices developed in our laboratory, are reported. In addition, the quality of the frozen-hydrated specimens are evaluated by analyzing the characteristics of the collected diffraction patterns. Based on the experimental results, the internal structures of the frozen-hydrated specimens and the future development for efficient diffraction data collection are discussed.

\section{Introduction}

Coherent X-ray diffraction imaging (CXDI) has been applied to visualize the internal structures of non-crystalline particles with dimensions of micrometers to sub-micrometers in materials science and biology (Miao et al., 1999, 2015). In CXDI experiments, a spatially isolated non-crystalline particle is irradiated by coherent X-rays, and then the Fraunhofer diffraction pattern of the particle is recorded by an area detector under the oversampling condition (Miao et al., 2003a). From the small-angle diffraction pattern, the electron density map of the particle projected along the direction of incident X-rays is, in principle, restored by applying the iterative phase-retrieval algorithm (Fienup, 1982). The long penetration depth of short-wavelength X-rays allows us to visualize the internal structures of whole specimen particles, which are opaque in transmission electron microscopy (EM), at resolutions higher than optical microscopy (Miao et al., 2002, 2003b, 2005; Robinson et al., 2001; Song et al., 2008; Nishino et al., 2009; Jiang et al., 2010; Takayama \& Nakasako, 2012; Nakasako et al., 2013; Gallagher-Jones et al., 2014).

Now, X-ray free-electron lasers (XFELs) are leading CXDI to a new stage. X-ray pulses with complete spatial coherence 
and duration of tens of femtoseconds are available at XFEL facilities such as the Linac Coherent Light Source (LCLS; Emma et al., 2010) and the SPring-8 Angstrom Compact FreeElectron Laser (SACLA; Ishikawa et al., 2012). We can collect diffraction data before radiation-damage processes occur (Chapman et al., 2006a; Hirata et al., 2014), although specimens irradiated by single XFEL pulses are destroyed at the atomic level (Chapman et al., 2006a; Nakasako et al., 2013). Owing to the repetition rate of XFEL pulses of $30-120 \mathrm{~Hz}$, a huge number of diffraction patterns can be collected within a short period of time.

In XFEL-CXDI structural studies, an important target is non-crystalline biological specimens such as cells and cellular organelles. In diffraction experiments, specimens are set in a vacuum to avoid absorption and scattering of the incident and diffracted X-rays by air. However, a vacuum environment causes fatal damage to biological specimens, which require aqueous environments to maintain their functional structures. In the XFEL-CXDI field, the liquid jet method (Kassemeyer, 2014), the aerosol method (Seibert et al., 2011; van der Schot et al., 2015) and the micro-liquid enclosure-array method (Kimura et al., 2014) have been developed to collect diffraction patterns from biological specimens in vacuum chambers of diffraction apparatus. To date, these experimental methods have been applied to the structure analyses of viruses (Seibert et al., 2011; Kassemeyer et al., 2012; Ekeberg et al., 2015), cellular components (Hantke et al., 2014) and bacterial cells (Kimura et al., 2014; van der Schot et al., 2015).

As an alternative approach to the three methods mentioned above, we proposed XFEL-CXDI experiments for frozenhydrated biological specimens at cryogenic temperatures (Nakasako et al., 2013; Oroguchi et al., 2015; Kobayashi et al., 2016). It has been demonstrated previously that cryogenic technologies enable us to store biological cells at low temperatures, for instance, in medical science (Chen, 1986; Medeiros et al., 2002; Schwartz, 1986). Frozen-hydrated cells and cellular organelles keep their functional structures (McDowall et al., 1983), and are still alive after returning to ambient temperatures (Gibson \& Khoury, 1986).

Cryogenic specimen preparation enables us to store frozenhydrated specimens in liquid nitrogen for CXDI experiments. Therefore, we can harvest a large amount of biological cells and isolated unstable cellular organelles at a desired period of the cell cycle. Moreover, frozen-hydrated specimens are free from adiabatic expansion, bubbling of water inside biological specimens, evaporation and drying even under vacuum conditions. The rate of XFEL pulses hitting single specimen particles can be controlled by the number density of particles dispersed on membranes as described below.

We have conducted a series of cryogenic XFEL-CXDI experiments for frozen-hydrated non-crystalline biological specimens, such as cells and cellular organelles, using our custom-made diffraction apparatus at SACLA (Fig. 1) (Nakasako et al., 2013; Oroguchi et al., 2015; Takayama et al., 2015; Kobayashi et al., 2016). In our XFEL-CXDI experiments, the specimen particles dispersed on thin membranes of specimen disks are flash-cooled into the frozen-hydrated state.

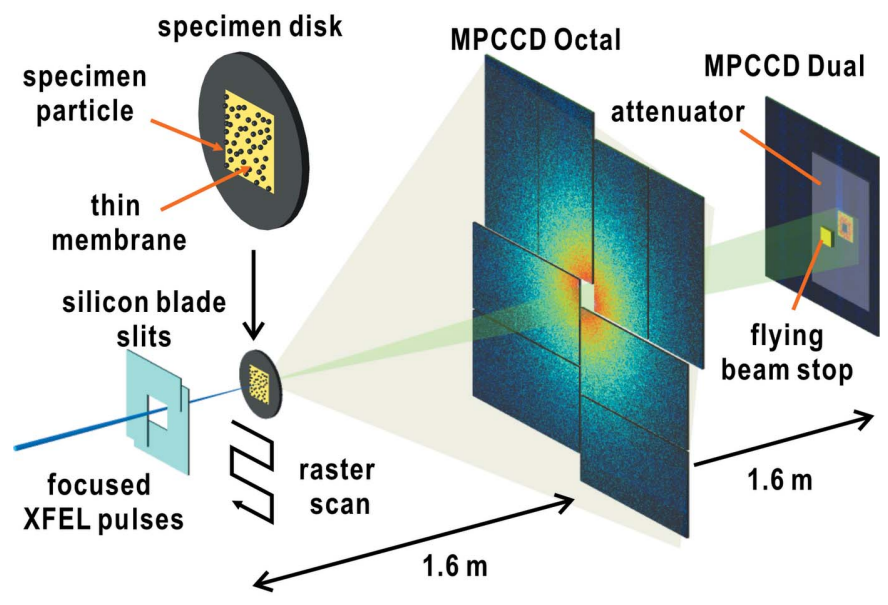

(a)

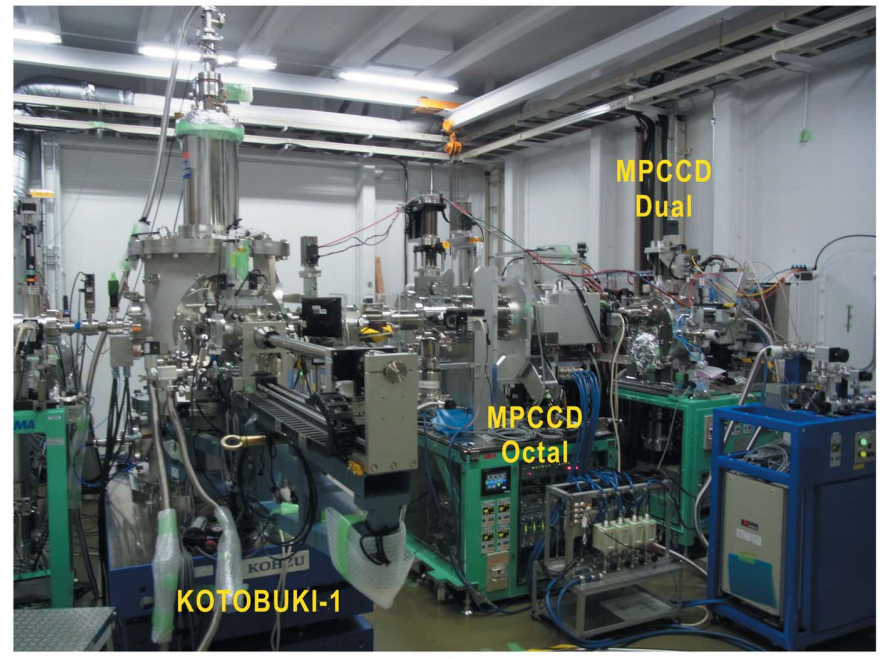

(b)

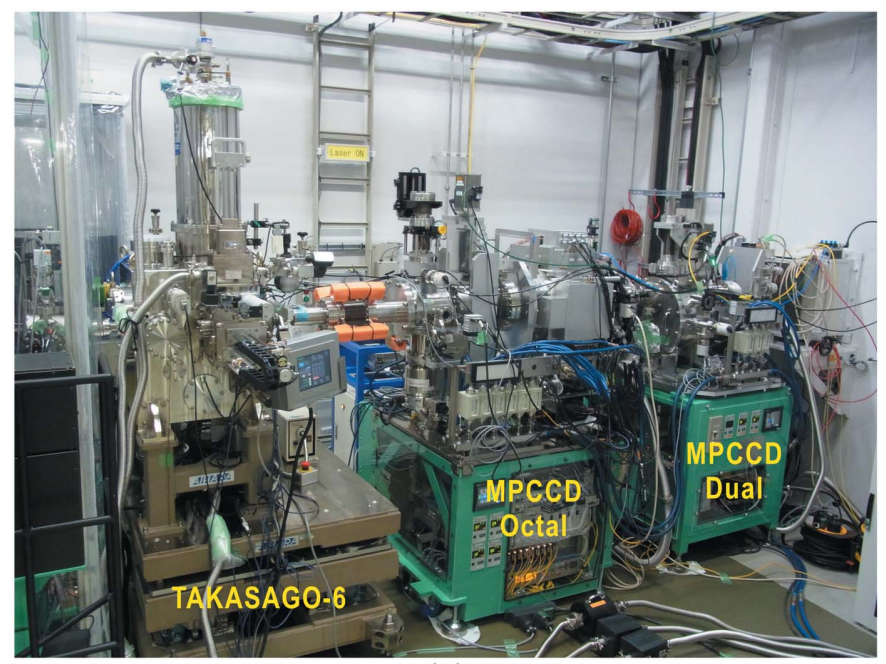

(c)

Figure 1

(a) Schematic illustration of cryogenic XFEL-CXDI experiments by raster scanning of specimen disks with non-crystalline specimen particles adsorbed onto the thin membrane region. Photographs of the KOTOBUKI-1 diffraction apparatus connected to the two MPCCD detectors in experimental hutch $3(b)$ and the TAKASAGO-6 with the two MPCCD detectors in hutch $4(c)$ of BL3 at SACLA. 
The specimen disks are then transferred into a vacuum chamber of the diffraction apparatus using specially designed devices. Focused single XFEL pulses destroy specimen particles at the atomic level and then particles within a diameter of approximately $10 \mu \mathrm{m}$ from the center of focused XFEL pulses are degraded or expelled from the membrane surfaces (Nakasako et al., 2013; Kobayashi et al., 2016). Therefore, diffraction patterns are collected through raster scanning specimen disks in $25 \mu \mathrm{m}$ steps to provide fresh specimen particles in the irradiation area (Nakasako et al., 2013; Kobayashi et al., 2016).

We have developed preparation procedures of frozenhydrated biological specimens for cryogenic XFEL-CXDI experiments by referring to the sophisticated specimen preparation procedures in cryogenic EM (Robarts \& Sleytr, 1985; Grassucci et al., 2007) and cryogenic protein X-ray crystallography (Nakasako, 1995). Here, we report the procedures including the materials and devices used, and evaluate the quality of the prepared specimens through analyzing the diffraction patterns from frozen-hydrated cyanobacterial cells. The methods described here would be useful for imaging the structures of non-crystalline particles in CXDI experiments.

\section{Methods}

\subsection{Membranes and specimen disks}

In our specimen preparation, specimen particles are dispersed on thin membranes attached to specimen disks under moist conditions. The specimen disks are flash-cooled by liquid ethane at a cooling rate of $\sim 3700 \mathrm{~K} \mathrm{~s}^{-1}$ (Nakasako, 1995). Therefore, the membranes and specimen disks are required to be able to withstand flash-cooling. In addition, diffraction intensities from the membranes must be negligibly weak in comparison with those from the specimen particles. To date, we have used molybdenum disks (Okenshoji, Japan) with single or multiple windows of carbon or chemically synthesized membranes (Fig. 2a). Because of their small thermal contraction, molybdenum disks are used in cryogenic EM (Grassucci et al., 2007). We have also used silicon disks with single or multiple silicon nitride $(\mathrm{SiN})$ membrane windows (Norcada, Canada), which are able to withstand flash-cooling by liquid ethane (Fig. $2 a$ ).

Carbon membranes of approximate thickness $30 \mathrm{~nm}$ are created on the surfaces of freshly cleaved mica plates of approximate size $5 \mathrm{~mm} \times 5 \mathrm{~mm}$ by using a JEE-420 vacuum evaporator (JEOL, Japan) (Fig. $2 b$ ). The thickness of the carbon membrane is controlled by monitoring the degree of carbon deposition on white filter paper placed near the mica plates. The carbon membranes are then detached from the mica plates at the air-water interface when inserting the carbon-deposited mica plates into water. The carbon membranes floating on the water are picked up by custommade molybdenum specimen disks (Fig. $2 a$ ) such that a single carbon film covers the windows without wrinkles.
Thermoset polymer membranes, hereafter designated as epoxy membranes, are chemically synthesized according to the previously published protocol (Watanabe \& Kunitake, 2007) (Fig. 2c). Poly[(o-cresyl glycidyl ether)-co-formaldehyde] (Sigma-Aldrich, USA) and polyethylenimine (SigmaAldrich, USA) are mixed in chloroform. A droplet of

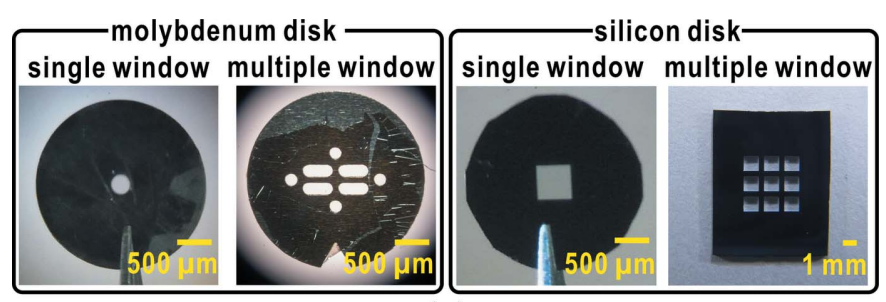

(a)

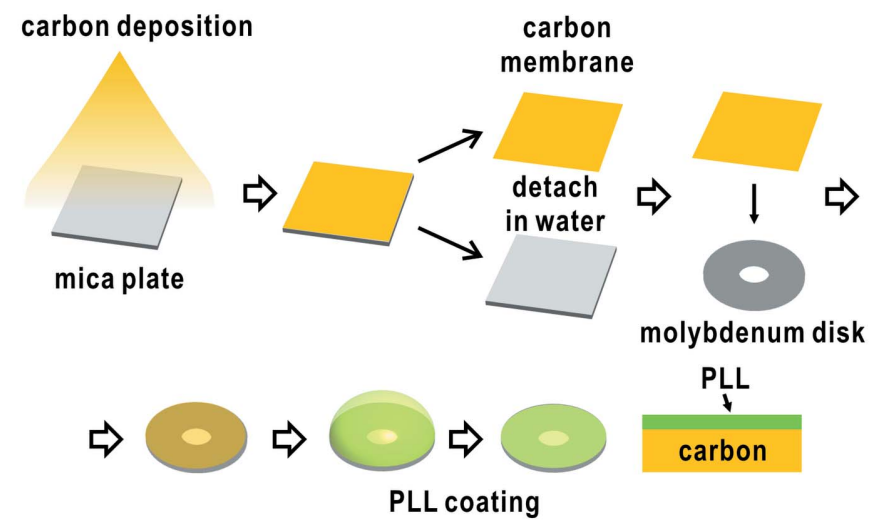

(b)

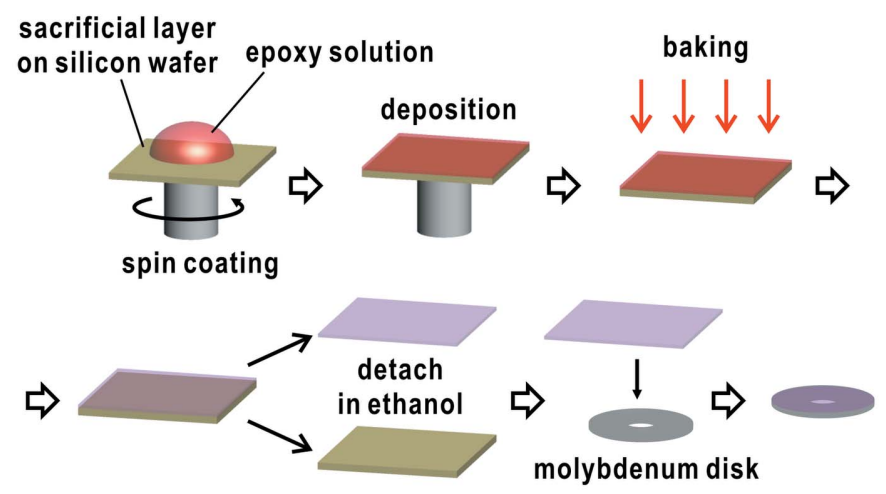

(c)

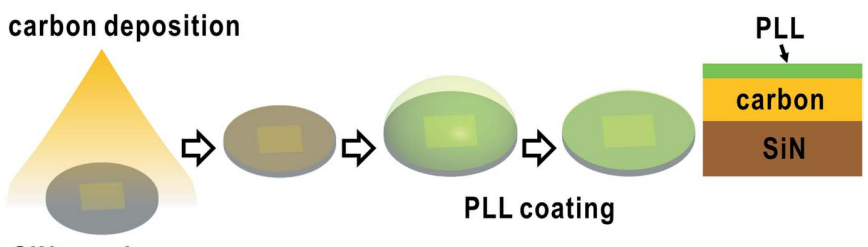

SiN membrane on silicon disk

(d)

Figure 2

(a) Two types of specimen disks used in our XFEL-CXDI experiments. Schematic illustrations on the preparation procedures of specimen disks with carbon membranes (b), epoxy membranes $(c)$ and $\mathrm{SiN}$ membranes $(d)$. 
the mixture is applied to a sacrificial layer of poly(4-hydroxystyrene) (Sigma-Aldrich, USA) prepared on a silicon wafer. To make a thin layer of the mixture, the wafer is rotated at a speed of 7500 r.p.m. for 1 min using a MS-A100 spin coater (Mikasa, Japan). Then, the wafer is baked at $393 \mathrm{~K}$ for $5 \mathrm{~min}$ in a BO-R65JB oven (Mitsubishi Electric, Japan). The thickness of the membrane can be controlled by the ratio of the weight of the polymers to the volume of chloroform in the range $0.1-1 \%(w / v)$, and is measured using an SP400 atomic force microscope (Seiko Instruments, Japan) and/or a MiniScope TM3000 scanning electron microscope (Hitachi High-Technologies, Japan). The sacrificial layer is dissolved in ethanol after being trimmed to an approximate size of $5 \mathrm{~mm} \times 5 \mathrm{~mm}$. Then, the epoxy membranes are detached from the silicon wafer. The epoxy membranes, having been transferred into water, are then picked-up by the molybdenum specimen disks (Fig. 2a).

In addition to the laboratory-made thin membranes, we use two types of silicon disks which have windows of $\mathrm{SiN}$ membrane of thickness $100 \mathrm{~nm}$ (Fig. 2a). One type is commercially available silicon disks of diameter $3 \mathrm{~mm}$ with one $500 \mu \mathrm{m} \times 500 \mu \mathrm{m}$ window (Norcada, Canada). On the other hand, we use custom-made silicon disks of size $10 \mathrm{~mm} \times$ $8 \mathrm{~mm}$ with nine windows of $1 \mathrm{~mm} \times 1 \mathrm{~mm}$ (Norcada, Canada) for collecting a huge number of diffraction patterns from the specimen particles.

Carbon membranes and $\mathrm{SiN}$ membranes are hydrophobic, while epoxy membranes have high affinity to biological specimens because of their hydrophilicity. Therefore, carbon membranes are treated with a $0.1 \mathrm{mg} \mathrm{mL}^{-1}$ solution of poly-Llysine (PLL) with a molecular weight of approximately $300 \mathrm{kDa}$ (Sigma-Aldrich, USA) to increase their affinity to biological specimens (Fig. 2b). After 30 min of treatment, unbound PLL is washed out using distilled water. Because SiN membranes have little affinity with PLL, they are coated with carbon layers of thickness $15 \mathrm{~nm}$ prior to the PLL decoration (Fig. 2d). The PLL treatment dramatically increases the affinity of these membranes to cells and organelles.

\subsection{Preparation of standard specimens}

In the cryogenic XFEL-CXDI experiments in this study, prior to diffraction data collection of biological specimens, we determine the camera parameters by analyzing diffraction patterns from single copper-oxide particles of cuboid shape (Kuo et al., 2007) through the protocol we proposed earlier (Sekiguchi et al., 2014a). A $2 \mu \mathrm{L}$ droplet of a concentrated suspension of copper-oxide particles is placed on the membranes using a micropipet (Fig. $3 a$ ). After adsorption of the copper-oxide particles to the membranes, the suspension is washed out. Finally, the membranes are dried under atmosphere. We select specimen disks with a larger number of isolated particles through surveying the membrane surfaces using a scanning electron microscope.

In addition, we use gold-colloidal particles (British Biocell International Solutions, UK) with diameters of $\sim 50 \mathrm{~nm}$ to measure the spatial coherence of focused X-ray pulses

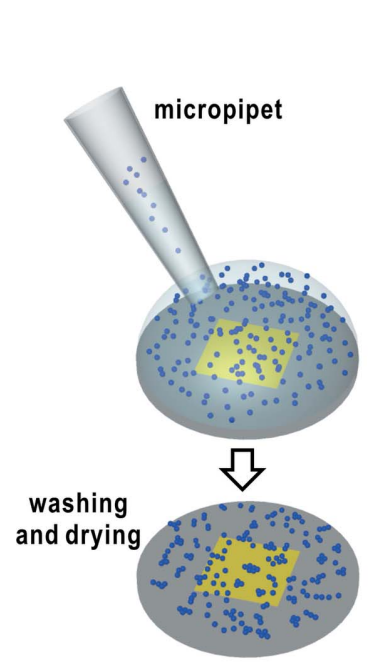

(a)

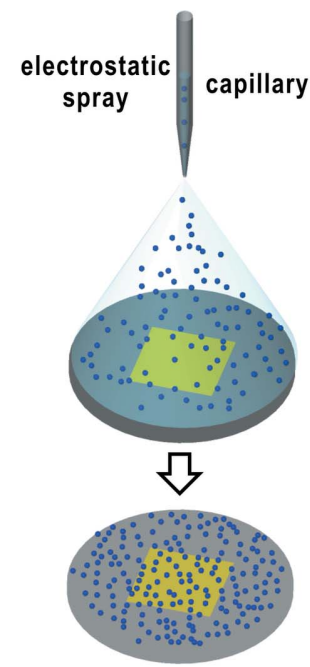

(b)

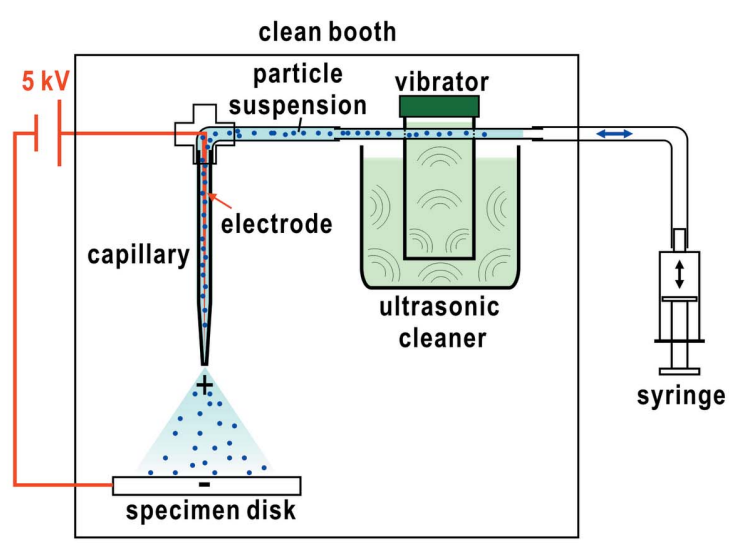

(c)

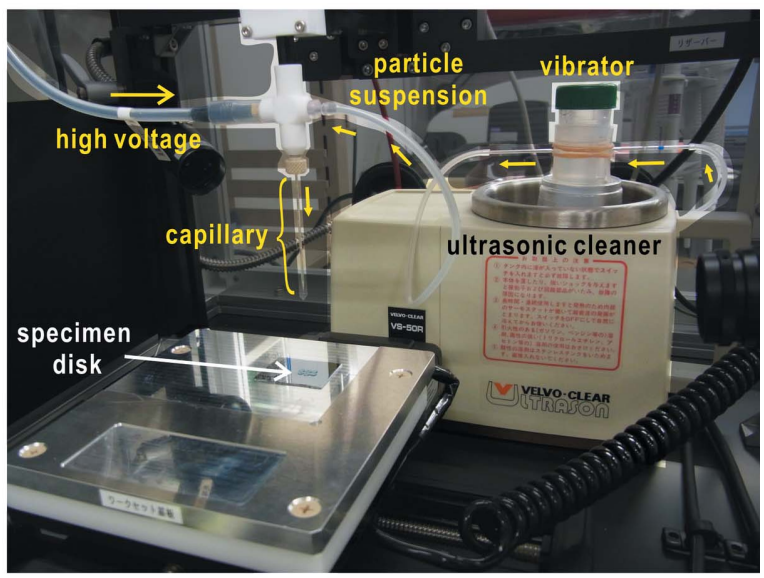

Figure 3

(d)

Schematic illustrations on the preparation of specimen disks carrying copper-oxide particles $(a)$ and gold-colloidal particles $(b)$. Schematic illustration $(c)$ and a photograph $(d)$ of our electrospray system used for uniformly dispersing gold-colloidal particles and PLL solutions. A thin electrode is inserted into a capillary tube with an inner diameter of 24 or $60 \mu \mathrm{m}$ filled with $8 \mu \mathrm{L}$ of concentrated suspension of gold-colloidal particles. The tip of the capillary nozzle is set about $10 \mathrm{~mm}$ apart from the specimen disk, and then the device is operated by applying a voltage of $5 \mathrm{kV}$ between the electrode and the base plate. The specimen suspension in the capillary tube is evacuated to the vibrator to be dispersed by the ultrasonic cleaner for every 10 min operation to prevent heavy clogging by gravity sedimentation at the tip of the capillary. 
through speckle visibility spectroscopy (Gutt et al., 2012; Lehmkühler et al., 2014). Details of the measurements and analytical methods for spatial coherence of XFEL pulses will be reported elsewhere.

We use the electrospray method (Oliver, 2004) to uniformly disperse the gold-colloidal particles on membranes without aggregates (Fig. $3 b$ ). In this method, a charged droplet of suspension is electrically driven from a thin capillary tube. Then, the charged droplet is divided into many small mist particles by electrostatic repulsion. Finally, the gold-colloidal particles are directly fixed on the membranes through evaporation and electric breakup of water from the mist particles.

The gold-colloidal particles of diameter $50 \mathrm{~nm}$ are dispersed on membranes using a PDS-D01 electrospray device (Hamamatsu Nano Technology, Japan) (Figs. 3b-3d). To avoid clogging of the gold-colloidal particles at the tip of the capillary tube of inner diameter $24 \mu \mathrm{m}$, the device is equipped with another custom-made device combining an ultrasonic cleaner and a syringe tube (Figs. $3 c-3 d$ ).

\subsection{Membranes with regularly arrayed PLL spots}

If specified areas on the carbon or carbon-coated SiN membranes are decorated with PLL, biological specimens are adsorbed only onto the PLL-decorated areas. To prepare such a membrane, we electrospray a $0.1 \mathrm{mg} \mathrm{ml}^{-1}$ PLL solution through a capillary tube of inner diameter $60 \mu \mathrm{m}$ onto the carbon or carbon-coated $\mathrm{SiN}$ membranes masked by a holey SiN plate of thickness $200 \mathrm{~nm}$ (Norcada, Canada) (Fig. 4a). The plate has regularly arrayed $3 \mu \mathrm{m}$-diameter pinholes with a period of $6.4 \mu \mathrm{m}$. On these membranes with regularly decorated PLL spots, biological particles are adsorbed only onto the spots after blotting off the suspension (Fig. 4b). In contrast, when a droplet of the suspension of biological particles is applied to the membranes decorated uniformly with PLL, the particles are adsorbed randomly onto the membranes (Fig. 4c).

\subsection{Preparation of frozen-hydrated biological specimens}

Fig. 5(a) shows a set of devices used for preparing frozenhydrated biological specimens. A humidity-controlling chamber is mounted on the specimen stage of an IX71 optical microscope (Olympus, Japan) (Takayama \& Nakasako, 2012). A HUM-1 humidity-controller (Rigaku, Japan) supplies moist air to the chamber. A custom-made freezing device is used to flash-cool specimens in liquid ethane. Through centrifugation, the concentrations of specimen particles in suspension are adjusted to yield finally a number density of approximately eight particles per $10 \mu \mathrm{m} \times 10 \mu \mathrm{m}$ area of the membrane.

A specimen disk caught at the tip of a precision pincette is set in a removable cassette holder of the humidity-controlling chamber (Fig. 5b). The interior of the cassette holder is filled with moist air from the HUM-1 controller. A $1-2 \mu \mathrm{L}$ droplet of specimen suspension is applied to the membrane of the disk under a relative humidity (RH) higher than $90 \%$. After a few minutes, sufficient for adsorption of specimen particles onto the membrane, an excess amount of suspension is blotted off using the tip of wick paper (MiTeGen, USA) or filter paper (Whatman, USA) under inspection by optical microscope.

When the number density of specimen particles and the amount of remaining buffer solution are within a desired range, the cassette holder is removed from the chamber. Then, we set the holder onto the freezing device, which is composed of a plunger block and a liquid-ethane bath (Fig. 5c). The RH inside the holder is kept higher than $90 \%$. Immediately after the pincette is fixed to the plunger block, the specimen disk at the tip of the pincette is dropped into the liquid-ethane bath. The flash-frozen specimen disks are stored in liquid nitrogen using disk containers (Yasuda Shoten, Japan) until used in the cryogenic XFEL-CXDI experiments (Fig. 5c). In the experiments, specimen disks are fixed to custom-made specimen holders with a pair of small neodymium magnets in order to be delivered from the liquid-nitrogen bath to the vacuum chamber of the diffraction apparatus (Fig. $5 d$ ). Four specimen disks of diameter $3 \mathrm{~mm}$ are set on an adaptor, and then a covering plate with four windows fixes the adaptor to the specimen holder. The large silicon specimen disk with nine SiN windows $(10 \mathrm{~mm} \times 8 \mathrm{~mm})$ is fixed to the specimen holder by a covering plate made of stainless steel attracted by the neodymium magnets.

In this study, we used a cyanobacterium as a representative biological specimen. Cyanobacteria of Prochlorococcus strain NIES-2087 were purchased from the National Institute for Environmental Studies, Japan. The size distribution of cyanobacteria in the PRO-99 culture medium (Moore et al., 2007) was measured by means of the dynamic light scattering (DLS) method by using a Zetasizer Nano S (Malvern, UK). The cyanobacterial cells of approximately $800 \mathrm{~nm}$ size are smaller than the focused spot sizes of the XFEL pulses (approximately 1-2 $\mu \mathrm{m}$ ) available at SACLA (Yumoto et al., 2013; Nakasako et al., 2013; Oroguchi et al., 2015).

\subsection{Cryogenic XFEL-CXDI experiments at SACLA}

Cryogenic XFEL-CXDI experiments were performed at BL3 of SACLA (Tono et al., 2013) using the KOTOBUKI-1 diffraction apparatus (Fig. 1b) (Nakasako et al., 2013; Sekiguchi et al., 2014b) or the TAKASAGO-6 diffraction apparatus (Fig. 1c) (Kobayashi et al., 2016). KOTOBUKI-1 is a prototype diffraction apparatus developed for examining the feasibility of cryogenic techniques introduced for CXDI experiments. The TAKASAGO-6 apparatus has been developed for high-throughput experiments based on the techniques developed using KOTOBUKI-1.

Each set of apparatus is equipped with a cryogenic pot on its goniometer inside the vacuum chamber. The temperature of the pot is kept at $66 \mathrm{~K}$ by the evaporation cooling effect (Nakasako et al., 2013; Kobayashi et al., 2016). Specimen disks fixed to specimen holders are transferred from a liquidnitrogen bath to the cryogenic pot using a carrier and an automated loading device without frosting and warming (Nakasako et al., 2013; Oroguchi et al., 2015; Kobayashi et al., 2016). 


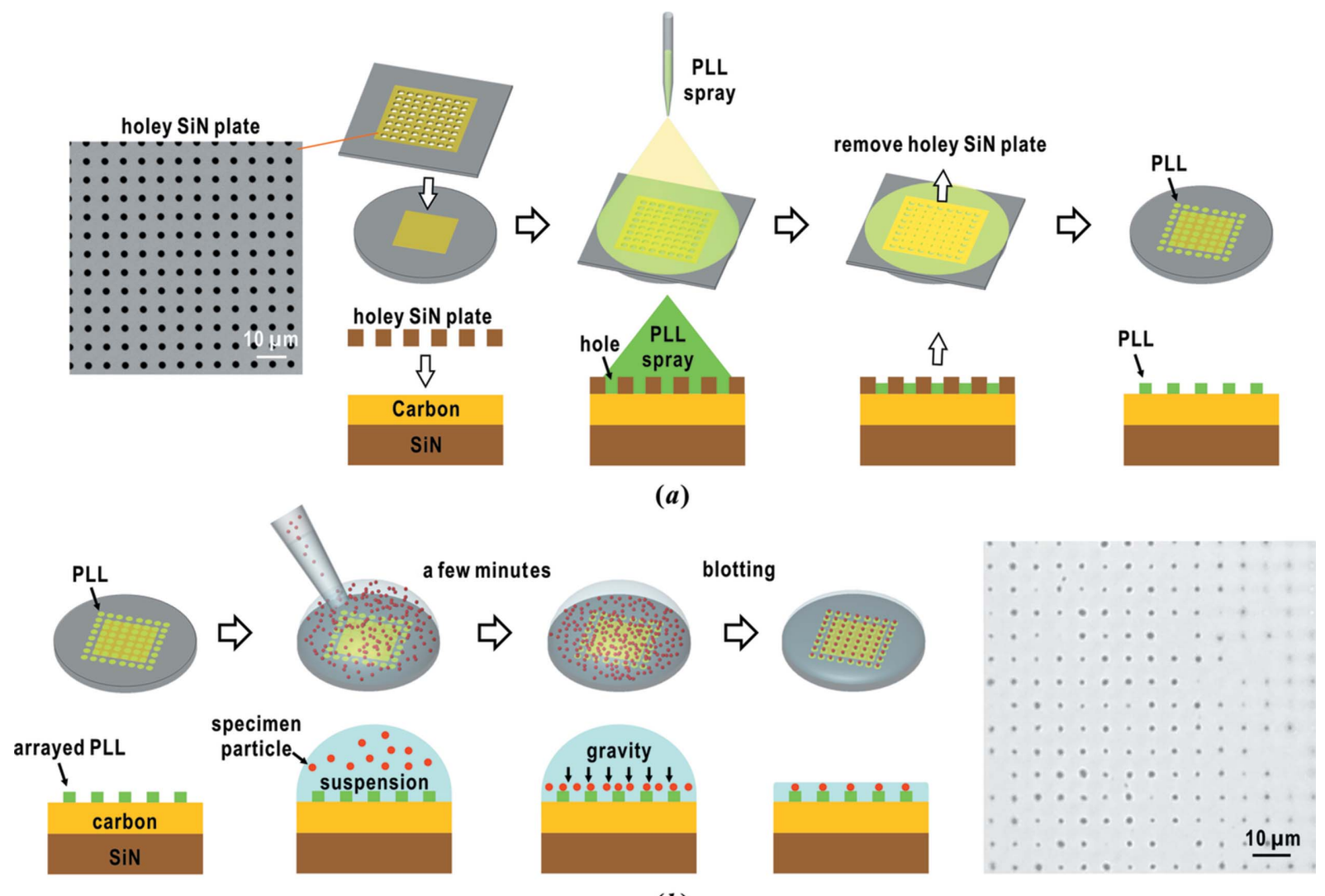

(b)
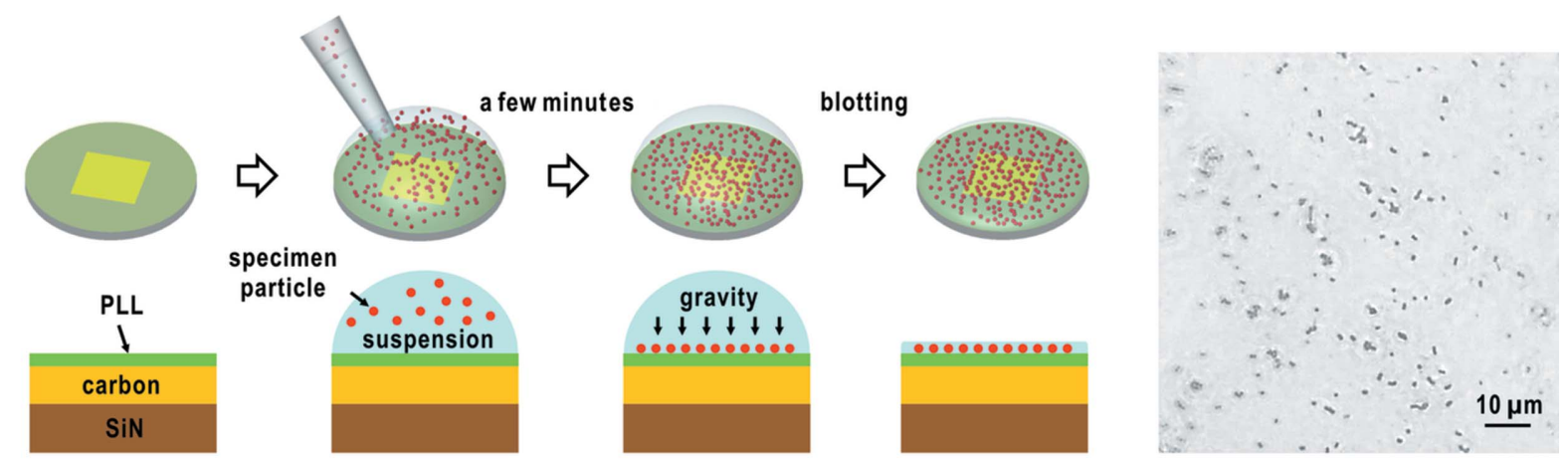

(c)

Figure 4

(a) Schematic illustration of the procedure to print regularly arrayed PLL spots onto carbon-coated SiN membranes by electrospraying the PLL solution with a commercially available holey SiN plate. The scanning electron micrograph of the holey SiN plate is displayed on the left (Norcada, Canada). Schematic illustrations of dispersing biological specimens, such as cyanobacteria cells on membranes with regularly printed PLL spots $(b)$ and with a uniform PLL layer $(c)$. The photographs on the right were taken after blotting by using the optical microscope IX71 in the phase-contrast mode.

$\mathrm{X}$-rays of wavelength $0.225 \mathrm{~nm}$ (X-ray photon energy of $5.5 \mathrm{keV}$ ) are focused to give intensities of $10^{10}-10^{11}$ photons $\mu \mathrm{m}^{-2}$ pulse ${ }^{-1}$ by focusing mirror optics (Yumoto et al., 2013). Each diffraction apparatus is placed so that the specimen position is within the beam waist of the focused X-ray pulses (Oroguchi et al., 2015; Kobayashi et al., 2016). Diffraction patterns are recorded by two multiport CCD (MPCCD) detectors (Kameshima et al., 2014) in a tandem arrangement (Fig. 1). A MPCCD Octal detector placed approximately $1.6 \mathrm{~m}$ downstream from the specimen position is composed of eight CCD sensor panels and records diffraction patterns in the resolution range approximately $7-210 \mathrm{~nm}$. The central aperture of the MPCCD Octal detector can be changed from a $3 \mathrm{~mm}$ square to a $9 \mathrm{~mm}$ square. A MPCCD Dual detector with two sensor panels is placed $3.2 \mathrm{~m}$ downstream of the specimen position with a beam stop of $2 \mathrm{~mm} \times 2 \mathrm{~mm}$. The Dual detector records small-angle diffraction patterns in the resolution range approximately $80-500 \mathrm{~nm}$ by attenuating the diffraction intensity using a set of aluminium films of thickness 15$100 \mu \mathrm{m}$. The small-angle resolution of approximately $500 \mathrm{~nm}$ 


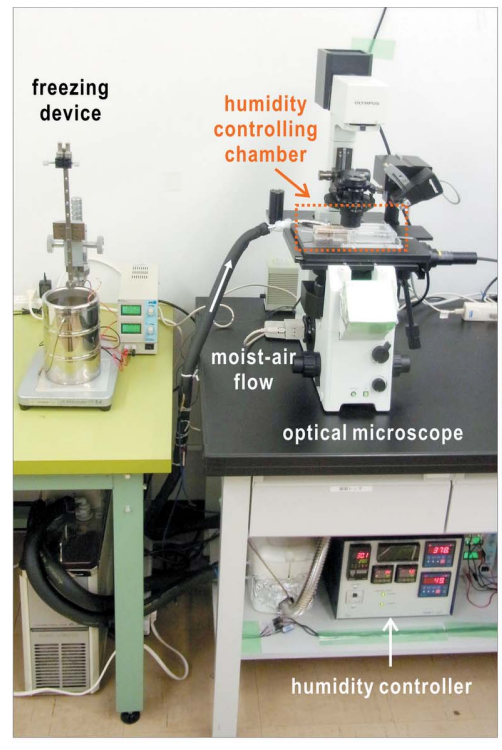

(a)

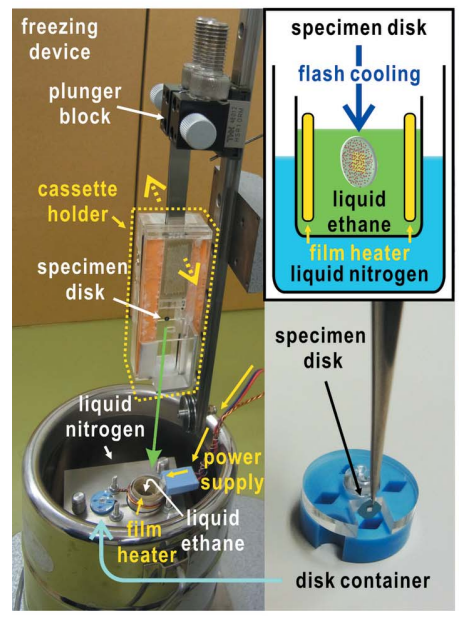

(c)

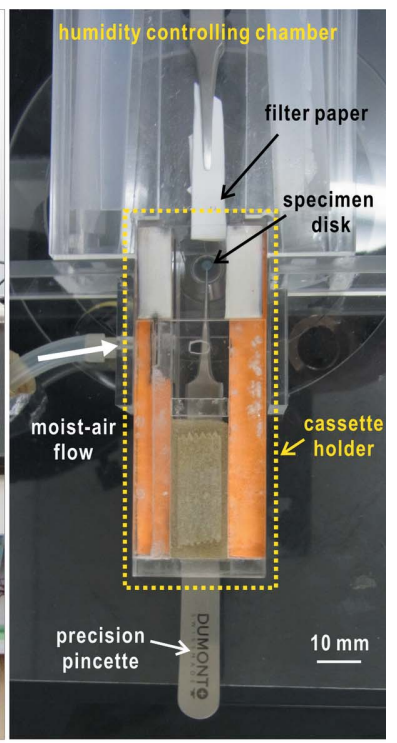

(b)

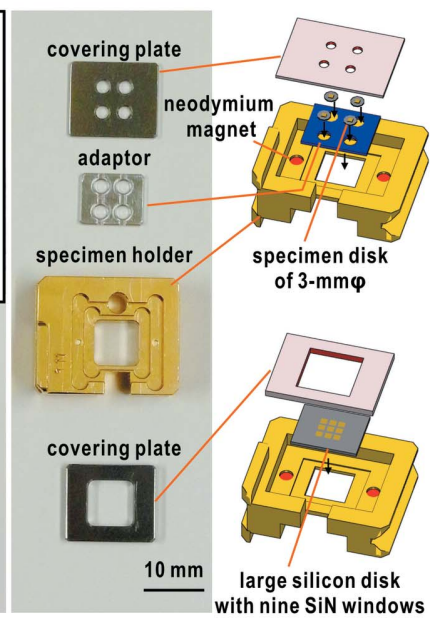

(d)
Figure 5

(a) Photograph of a set of devices used for preparing frozen-hydrated biological specimens, and a custom-made freezing device. (b) Magnified view of the humidity controlling chamber. A cassette holder is filled with moist air from the HUM-1, and is removable from the main body of the chamber. (c) Photograph of the flash-freezing device just before plunging the specimen disk into the liquid-ethane bath. Liquid ethane is produced by blowing ethane gas onto the wall of an aluminium cap cooled by liquid nitrogen. The temperature of liquid ethane is kept above the melting point by using a film heater of $7 \Omega$ (Sakaguchi Dennetsu, Japan) connected to a power supply of $24 \mathrm{~V}$ as schematically illustrated in the inset on the upper right. The inset on the lower right is a photograph of a disk container to store flash-frozen specimen disks in liquid nitrogen (Yasuda Shoten, Japan). (d) Specimen holder and plates used to bring specimen disks from the liquid-nitrogen bath to the vacuum chambers of diffraction apparatus. Plates shown in the left photograph are used as illustrated on the right. Two neodymium magnets of $1.5 \mathrm{~mm}$ diameter and $3 \mathrm{~mm}$ height are buried in the specimen holder. An aluminium adaptor for carrying four specimen disks of diameter $3 \mathrm{~mm}$ is fixed to the specimen holder by a covering plate with four holes. The other covering plate is used to fix a silicon specimen disk with nine SiN windows to the specimen holder. can be achieved through eliminating the parasitic scattering from upstream optics by adjusting the positions of siliconblade slits (Oroguchi et al., 2015).

Diffraction patterns are collected through raster-scanning the specimen disks in steps of $25 \mu \mathrm{m}$ in order to provide fresh specimen particles in the irradiation area. By using the highprecision goniometer of the prototype KOTOBUKI-1 apparatus, specimen disks of $3 \mathrm{~mm}$ diameter (Figs. $2 a$ and $5 d$ ) are raster-scanned synchronously with the motion of a pulse selector upstream of the beamline at a repetition rate of either $1 \mathrm{~Hz}$ or $2 \mathrm{~Hz}$. The high-speed translation stage of the TAKASAGO-6 apparatus enables us to raster-scan the specimen disks for every XFEL pulse provided at a repetition rate of $30 \mathrm{~Hz}$. We can utilize both large disks with nine $\mathrm{SiN}$ windows and disks of $3 \mathrm{~mm}$ diameter (Figs. $2 a$ and $5 d$ ) when using TAKASAGO-6.

\subsection{Data processing and structure analysis}

A large number of diffraction patterns collected in each raster scan are processed by the G-SITENNO suite (Sekiguchi et $a l ., 2014 a, b)$ on the SACLA HPC supercomputer system (Joti et al., 2015). The G-SITENNO suite automatically subtracts dark current of the detectors from diffraction patterns, selects diffraction patterns with good signal-to-noise ratios at a desired resolution, and merges diffraction patterns recorded by the two MPCCD detectors. In addition, the suite preliminary provides projection electron density maps of the specimen particles by applying the phase-retrieval algorithm to selected diffraction patterns (Oroguchi \& Nakasako, 2013) on the Mini-K supercomputer system (Joti et al., 2015). After the data processing, G-SITENNO reports statistics of the diffraction data sets, and provides a compiled summary of diffraction patterns and retrieved projection images.

Further structure analyses are conducted for selected highquality diffraction patterns of single particles which show good signal-to-noise ratios and maximum resolutions. The analyses are carried out using our structure analysis scheme ASURA (Sekiguchi et al., 2016) on the Mini-K supercomputer system. In the first step, we determine the most probable overall shape of a particle, i.e. the support, by using the hybrid-input-output algorithm (Fienup, 1982) and the shrink-wrap algorithm (Marchesini et al., 2003) according to the procedure reported previously (Kodama \& Nakasako, 2011; Oroguchi \& Nakasako, 2013). In the next step, the most accurate electron density distribution inside the determined support is estimated by applying the oversampling-smoothness algorithm (Rodriguez et al., 2013). In each step we objectively select the most probable electron density maps by applying multivariate analysis to approximately 1000 electron density maps retrieved starting from different initial densities. Finally, we calculate a map as the average of the most probable class in the multivariate analysis. The effective resolution of the projection map is estimated by the phase-retrieval transfer function (PRTF) (Chapman et al., 2006b; Oroguchi et al., 2015; Sekiguchi et al., 2016). 
When diffraction patterns lose a lot of speckles around the zero-diffraction angle because of saturation of the CCD detectors, we apply the dark-field phaseretrieval algorithm under the constraint of central symmetry in diffraction patterns (Kobayashi et al., 2014).

\section{Results}

\subsection{Handling of specimen disks}

The three types of membranes used in this study are capable of withstanding flash-cooling. Carbon and epoxy membranes on molybdenum specimen disks of diameter $3 \mathrm{~mm}$ (Fig. 2a) are mainly used for preliminary examination of the specimens. In contrast, the large silicon disks with nine windows (Fig. 2a) are suitable for collecting a large number of diffraction patterns of specimens, the preparation conditions of which are well established.

In addition, the simplified procedure utilizing specimen holders with the pair of magnets, adaptor and covering plate is advantageous for avoiding problems such as the destruction of membranes by the tip of the pincette (Fig. $5 d$ ). Mounting four small specimen disks on the specimen holder by use of the adaptor takes approximately $5 \mathrm{~min}$ in liquid nitrogen. The large SiN specimen disk is fixed to the specimen holder within $15 \mathrm{~s}$.

\subsection{Diffraction patterns from membranes}

Diffraction patterns from the three types of membranes are compared with parasitic scattering from the upstream optics after tuning the positions of a pair of slits and the beam stop (Fig. 6a). Diffraction intensities from carbon and $\mathrm{SiN}$ membranes are approximately less than five X-ray photons per pixel in smallangle regions around $3 \mu \mathrm{m}^{-1}$, and almost invisible beyond $5 \mu^{-1}$ (Figs. $6 b$ and $6 c$ ). Some detector pixels in the smallangle region close to the beam stop received 20 photons per pixel. However, the diffraction intensity is negligibly small in comparison with that from biological specimens exceeding $10^{5}$ $\mathrm{X}$-ray photons per pixel. When recording diffraction patterns of the biological specimens, an aluminium attenuator placed in front of the MPCCD Dual detector completely absorbs the weak diffraction from the membranes as well as parasitic scattering. Carbon and $\mathrm{SiN}$ membranes occasionally crack over exposed windows by Coulomb explosion of particles on the membranes. These narrow and long cracks give strong and

Figure 6

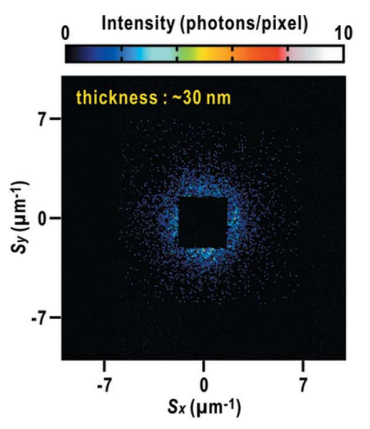

(b)

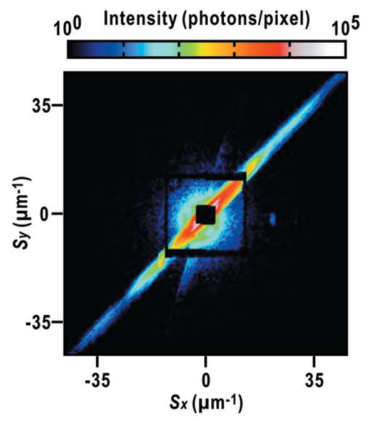

(d)

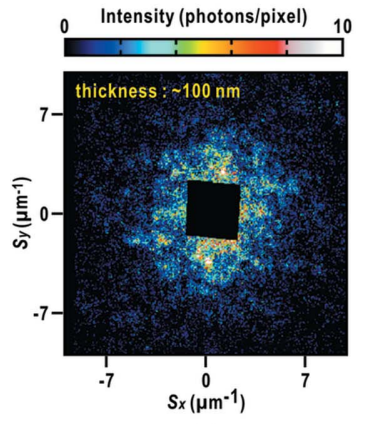

(e)

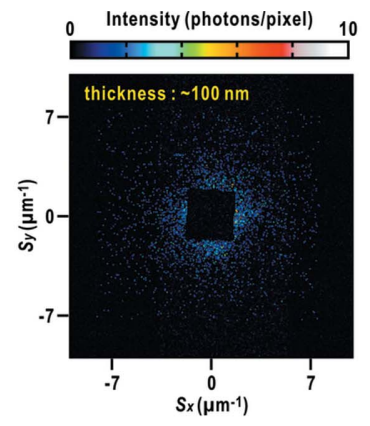

(c)
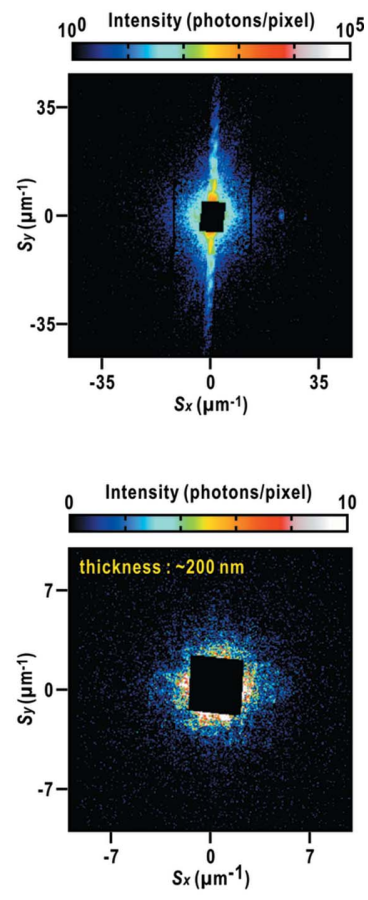

stop coming from optics upstream. Diffraction patterns from a carbon membrane of thickness $\sim 30 \mathrm{~nm}(b)$ and a SiN membrane of thickness $\sim 100 \mathrm{~nm}(c) . S$ is the scattering vector and defined as $S=2 \sin \theta / \lambda$, where $2 \theta$ is the diffraction angle and $\lambda$ is the X-ray wavelength. $(d)$ Typical patterns from cracked membranes. Each pattern is width of each slit is estimated to be approximately $90 \mathrm{~nm}$ (left panel), $100 \mathrm{~nm}$ (center) and $300 \mathrm{~nm}$ (right). (e) Diffraction patterns from epoxy membranes with thickness of $\sim 20 \mathrm{~nm}$ (left panel), $100 \mathrm{~nm}$ (center) and $200 \mathrm{~nm}$ (right).

anisotropic streak patterns (Fig. 6d). The tendency of cracking is likely prominent when the number density of particles is high.

Diffraction patterns from epoxy membranes vary depending on their thickness (Fig. 6e). Epoxy membranes as thin as $20 \mathrm{~nm}$ give speckle peaks in the small-angle region up to a resolution of $8 \mu \mathrm{m}^{-1}$. The speckle peaks suggest the presence of fluctuations in electron densities with approximate sizes of $200 \mathrm{~nm}$, probably originating from planar micro domains in the epoxy polymers. On the other hand, speckle patterns become weak in membranes of thickness $100 \mathrm{~nm}$. When the thickness of the membranes reaches $200 \mathrm{~nm}$, speckle patterns disappear, indicating that the electron density projected along the incident X-rays becomes uniform by the increase in the thickness of the films. Therefore, we pay attention to controlling the thickness of epoxy membranes by 
adjusting the amount of reagents. In contrast to carbon and $\mathrm{SiN}$ membranes, epoxy membranes rarely crack even when particles are densely distributed in the irradiation area.

\subsection{Standard specimens}

It is difficult to apply the electrospray method to the suspension of copper-oxide particles, because the stabilization buffer contains a high concentration of reagents $(33 \mathrm{~m} M$ sodium dodecyl sulfate, $200 \mathrm{~m} M$ sodium ascorbate and $1 M$ sodium hydroxide). When the suspension of copper-oxide particles is electrosprayed, powders of the three reagents appear on the membranes due to the evaporation and electric breakup of water from the sprayed mist of the suspension. Thus, we abandoned the application of the electrospray method for the suspension of copper-oxide particles. However, even from specimens containing aggregates of copper-oxide particles as identified in the scanning electron microscopy (SEM) images (Fig. 7a), diffraction patterns from single copper-oxide particles (Fig. $7 a$ ) are stochastically obtained at a
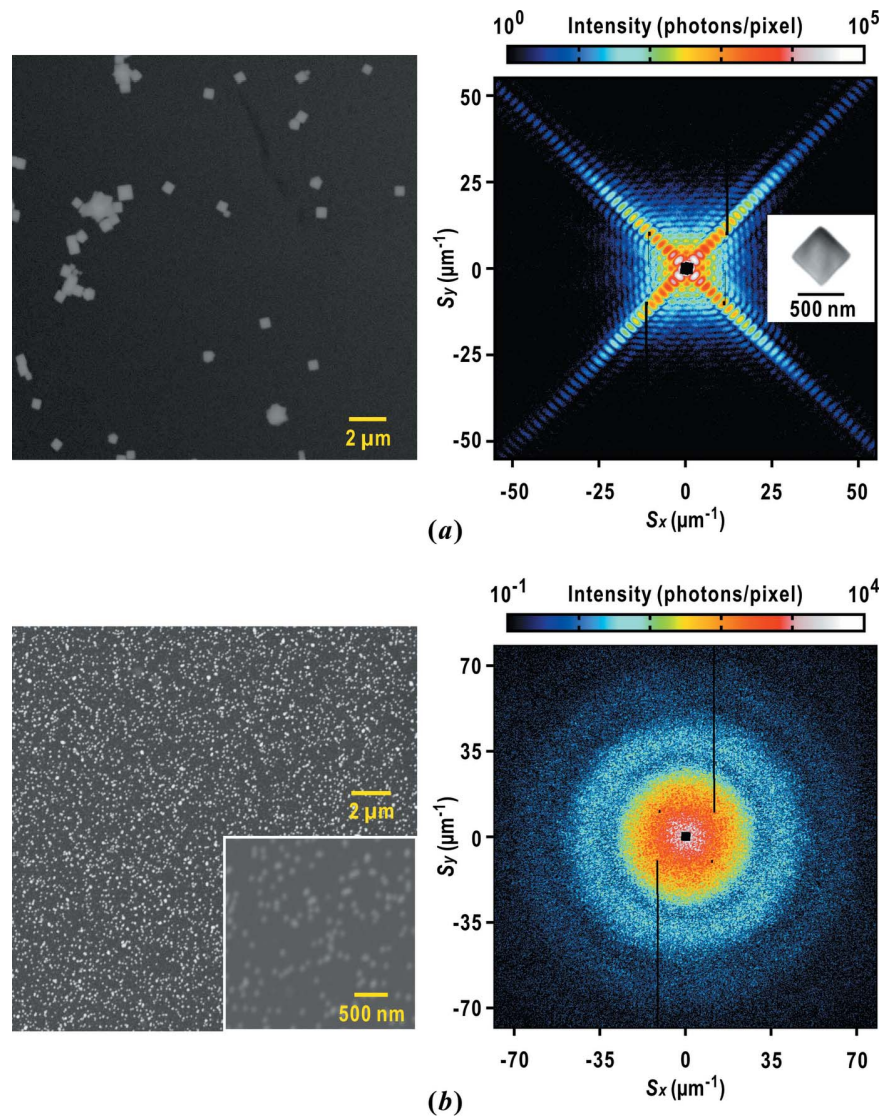

Figure 7

Preparation of standard specimens of metal particles. (a) SEM image of dispersed copper-oxide particles (left panel), and a diffraction pattern from a single copper-oxide particle of approximate size $500 \mathrm{~nm}$. The electron density map projected along the direction of the incident X-ray pulse is retrieved as shown in the inset. We applied the ASURA protocol to the diffraction pattern up to a resolution of $25 \mathrm{~nm}$ at edge (Table 1). (b) SEM image and diffraction pattern of gold-colloidal particles of diameter $50 \mathrm{~nm}$ densely dispersed on a thin membrane. rate of $11 \%$ against the total number of hits of XFEL pulses on single or clusters of particles during a raster scan. This efficiency is sufficient to determine the camera parameters.

For measuring the spatial coherence of the focused X-ray beam through visibility spectroscopy, specimen disks with uniformly and densely dispersed small gold-colloidal particles are necessary. The gold-colloidal particles in distilled water are uniformly dispersed without aggregates by using the electrospray device (Fig. 7b). The diffraction patterns are composed of concentric rings, which originate from the overall shape of the gold-colloidal particles. The small speckle peaks originate from the interference of X-rays diffracted by particles in the irradiation area.

It takes 20 min until the number density of sprayed particles reaches 40 particles $\mu \mathrm{m}^{-2}$. In addition, the custom-made device is useful for preventing clogging caused by gravity sedimentation of the gold-colloidal particles (Figs. $3 c$ and $3 d$ ).

\subsection{Diffraction patterns from frozen-hydrated cyanobacterial cells}

The efficiency of diffraction data collection relies on the number density and dispersity of specimen particles. In the case of frozen-hydrated cyanobacterial cells, the average number density of approximately 8 particles per $100 \mu \mathrm{m}^{2}$ results in a hit rate of approximately $35 \%$ against the total number of incident X-ray pulses. On the other hand, the quality of diffraction patterns depends on the amount of suspension remaining on the membranes (Fig. 8).

Fig. 8(a) displays a representative diffraction pattern from single cyanobacterial cell specimens with a diminished amount of suspension. The probability of obtaining such diffraction patterns is a few percent against the total number of XFEL pulses used in the raster scans. These patterns are composed of speckle peaks with sizes of $\sim 1.3 \mu \mathrm{m}^{-1}$. They are comparable with the reciprocal sizes of single cyanobacterial cells of $\sim 780 \mathrm{~nm}$. Among the diffraction patterns obtained through raster scans, the total diffraction intensities in the area displayed in Fig. 8(a) $\left(40 \mu^{-1}\right.$ at the edge $)$ is distributed from $10^{5}$ to $10^{8}$ photons, and the most frequently observed maximum resolution is $\sim 50 \mathrm{~nm}$.

The diffraction pattern from a semi-dried cyanobacterial cell has an ellipsoid shape with long and short axes of approximately $300 \mathrm{~nm}$ and $90 \mathrm{~nm}$, respectively (Fig. $8 b$ ). The size is less than half of that shown in Fig. 8(a). In addition, the shape and size are inconsistent with those observed for Prochlorococcus strain MIT9313 in EM (Grassucci et al., 2007). The statistics for the diffraction patterns collected in raster scans are different from those of the specimens shown in Fig. 8(a). The total diffraction intensities are of the order of $10^{4}$ photons, and the most frequently observed maximum resolution is approximately $100 \mathrm{~nm}$. These findings suggest that evaporation of water would cause shrinkage of the cells and decrease the total cross section for X-rays.

When an excess amount of specimen suspension remains on the membranes, diffraction patterns are composed of speckles 

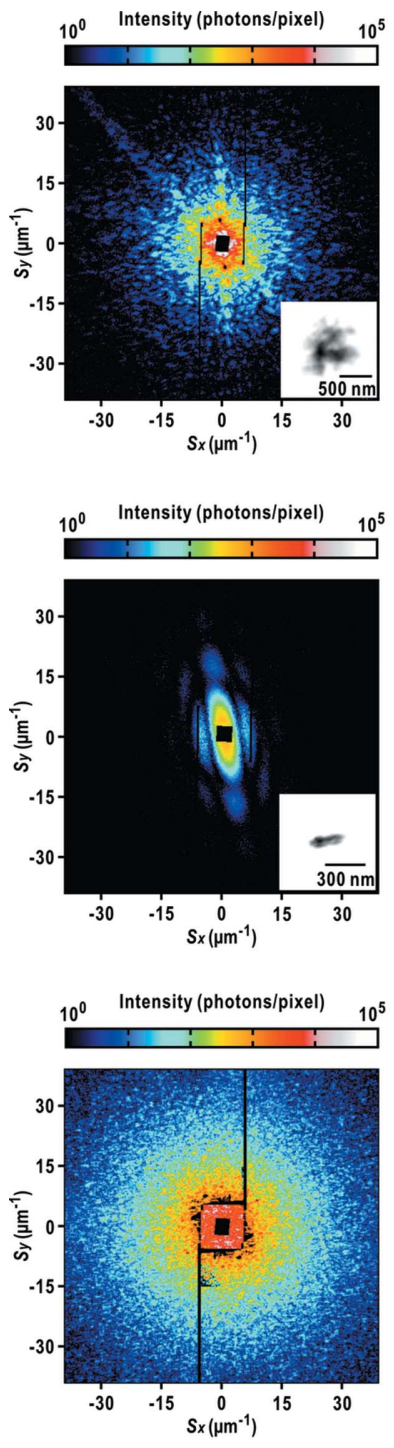

Figure 8

Diffraction patterns from flash-cooled cyanobacteria specimens with the frequency distribution regarding the total diffraction intensities and maximum resolutions. The total intensity of each diffraction pattern was calculated over the area displayed. The maximum resolution is defined as the highest-resolution shell including at least three detector pixels with more than four photons (Sekiguchi et al., 2014b). We did not analyze diffraction patterns with less than three detector pixels, which count more than four photons. The data shown are those for cyanobacteria surrounded by an appropriate amount of buffer solution $(a)$, semi-dried $(b)$ and heavily wet $(c)$. The electron density maps retrieved from the diffraction patterns in Figs. 8(a) and $8(b)$ are shown in the inset. We applied the ASURA protocol to these diffraction patterns up to a resolution of $50 \mathrm{~nm}$ at edge. The statistics of the retrieved electron density maps are listed in Table 1.

with smaller sizes than one-third of the reciprocal sizes of cyanobacterial cells (Fig. 8c). Due to the large cross section in the irradiation area, most diffraction patterns have total diffraction intensities that are 10-1000 times higher than those from single cyanobacterial cells with a thin layer of suspension in Fig. 8(a), and then the maximum resolution of diffraction patterns reaches $20 \mathrm{~nm}$. Taking the sizes of the object expected from speckle sizes, cells would overlap along the direction of the incident X-rays. A non-uniform thickness of vitreous ice within the irradiation area would also contribute to the diffraction patterns.
3.5. Projection electron density maps of cyanobacterial cells

Here we show diffraction patterns of flash-frozen cyanobacterial cells on a large SiN membrane. Through raster scans of 14512 points, we selected 63 diffraction patterns, such as those shown in Figs. 9(a) and 9(b), as being suitable for structure analyses with respect to the speckle size, diffraction intensities and maximum resolutions. The evaluated centro-symmetry scores of the diffraction patterns are better than 0.8 (Table 1), and then the speckle peaks of sizes $1.2-1.4 \mu^{-1}$ are visible beyond resolutions of $20 \mu \mathrm{m}^{-1}$. The isotropic distribution of speckle patterns suggests globular shapes of the frozen-hydrated cyanobacterial cells.

Through the $A S U R A$ protocol, the 63 projection electron density maps were retrieved at effective resolutions of better than $150 \mathrm{~nm}$ (Figs. $8 a, 9 a, 9 b$ and Table 1). Fig. 9(c) shows the distribution of cellular size measured for electron density regions higher than 0.25 . The distribution has a peak centered at $775 \mathrm{~nm}$ with a standard deviation of $38 \mathrm{~nm}$ (Fig. 9c). The peak value agrees with the cell size estimated by the DLS measurement prior to the specimen preparation $(780 \pm 230 \mathrm{~nm})$. In addition, both results are consistent with the cell sizes observed by optical microscopy (Fig. 9d). These findings indicate that the sizes of flash-frozen cyanobacterial cells would remain unchanged from those at ambient temperature.

In the phase-retrieved maps of cyanobacterial cells (Figs. $9 a$ and 9b), the electron densities inside the cells vary in the range $0.4-1.0$, while those in the border regions of overall shapes are in the range $0.3-0.4$. In particular, highelectron-density C-shaped regions with approximate sizes of $500 \mathrm{~nm}$ are sometimes observed. The $\mathrm{C}$-shaped regions can be assigned as the stack of thylakoid membranes with reference to the tomography study of a frozen-hydrated cyanobacterial cell using cryogenic transmission EM (Ting et al., 2007).

\subsection{Diffraction data collection for specimen disks with regularly arrayed specimen particles}

We compared the efficiency of diffraction data collection between specimen disks with regularly arrayed particles (Fig. 4b) and those with randomly dispersed specimen parti- 
cles (Fig. 4c). For randomly dispersed specimens with a number density of approximately 8 particles per $100 \mu \mathrm{m}^{2}$, $1.2 \%$ of the total incident XFEL pulses yielded diffraction patterns suitable for structure analyses such as shown in Figs. $8(a), 9(a)$ and $9(b)$. In contrast, specimen disks with regularly arrayed particles recorded a hit rate of $2.4 \%$. This difference is significant enough to increase the number of diffraction patterns suitable for structure analyses when millions of XFEL pulses are available in a beam time.

We rarely observed diffraction patterns from heavily wet specimens such as those shown in Fig. 8(c) when specimen disks with regularly arrayed PLL spots were used, probably because the surfaces of those membranes, except for the PLL spots, are hydrophobic to prevent them from wetting. In addition, the hydrophobic surfaces help us to easily remove the excess amount of suspension by blotting before flashcooling. Therefore, specimen disks with regularly arrayed particles would be advantageous for collecting high-quality diffraction patterns from well characterized specimen particles. In contrast, because it is easy to prepare membranes uniformly decorated by PLL, specimen disks with randomly dispersed specimen particles are suitable for preliminary trials of collecting a large number of diffraction patterns.

\subsection{Diffraction from ice crystals of sub-micrometer size}

If frosting occurs during the handling of cooled specimens (Fig. $5 c$ ) or the temperature of specimens rises beyond the glass transition temperature of the specimen buffer (probably 150-180 K), a lot of hexagonal or cubic ice crystals appear causing intense streak patterns as shown in Fig. 10. We observed streak patterns quite rarely at a probability of less than $0.003 \%$ during our experiments in 2014 and 2015. Therefore, the procedures described here enable us to prepare frozen-hydrated biological specimens almost free from ice crystals.

(a)
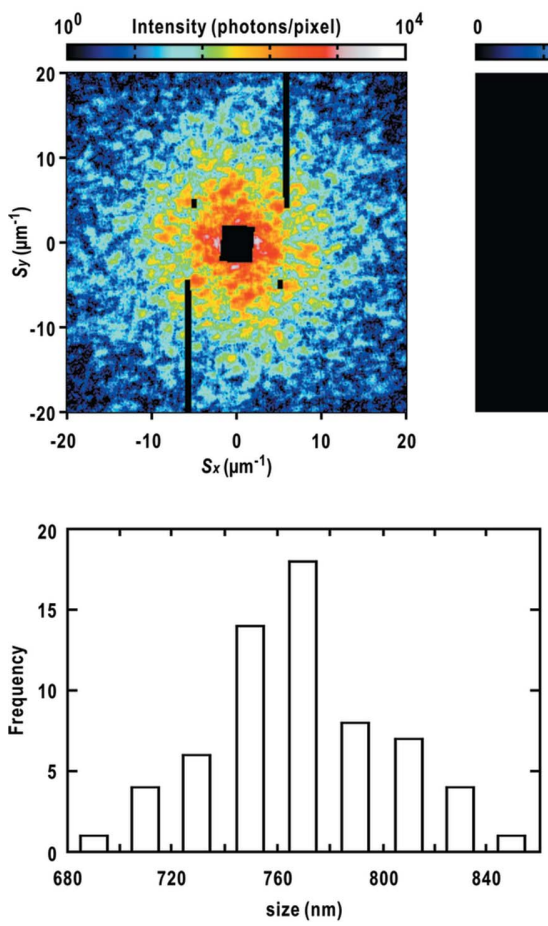

(c)

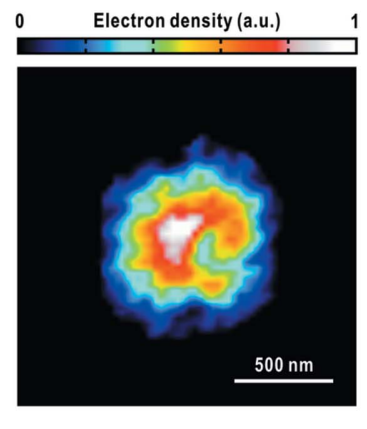

(b)

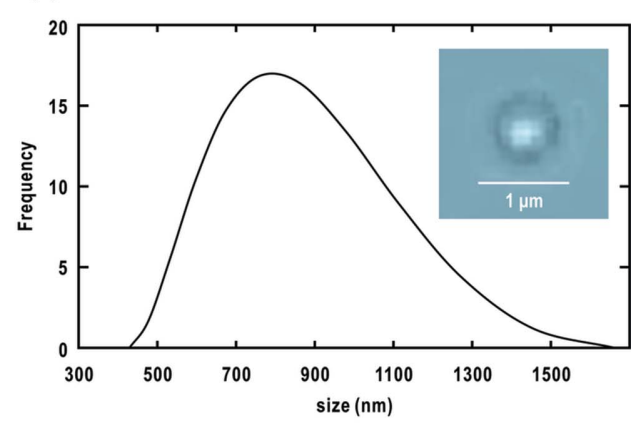

(d)

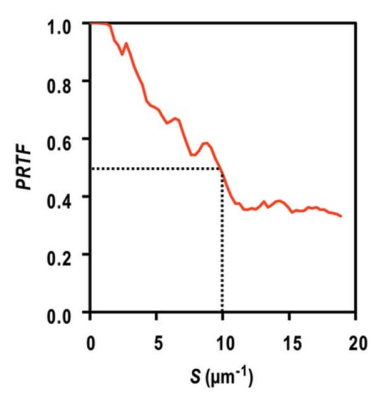

Figure 9

Two representative examples of diffraction patterns and the most probable electron density maps of frozen-hydrated single cyanobacterial cells. We applied the ASURA protocol to the diffraction patterns up to a resolution of $50 \mathrm{~nm}$ at edge. The statistics of the diffraction patterns and phaseretrieved projection electron density maps are summarized in Table 1 . The effective resolutions of the density maps are estimated by using the PRTF. Size distribution of cyanobacterial cells for 63 retrieved projection electron density maps $(c)$, and that measured by DLS $(d)$. The inset shows an image of a cyanobacterial cell taken using an IX71 optical microscope.

\section{Discussion}

We have developed membranes, devices and procedures to prepare frozenhydrated biological specimens and standard metal specimens for cryogenic XFEL-CXDI experiments. In addition, the quality of the prepared specimens was examined through diffraction experiments. Based on the present results, here we discuss future developments in specimen preparation, and the characteristics of frozen-hydrated biological specimens in CXDI experiments.

\subsection{Future development in specimen preparation}

As demonstrated in Fig. 8, an appropriate amount of buffer solution remaining around specimen particles is one of the important factors for collecting diffraction patterns suitable for structure analyses. Control of the amount of suspension through blotting depends on the contact modes of wick papers or filter papers with droplets of suspension, such as shape, period and 
Table 1

Statistics of diffraction patterns and phase-retrieved electron density maps.

\begin{tabular}{|c|c|c|c|c|c|}
\hline & Fig. 7(a) & Fig. 8(a) & Fig. 8(b) & Fig. 9(a) & Fig. $9(b)$ \\
\hline$C_{\mathrm{sym}}^{\dagger}$ & 0.72 & 0.85 & 0.46 & 0.86 & 0.81 \\
\hline Maximum resolution $(\mathrm{nm}) \ddagger$ & 11.1 & 20.0 & 50.1 & 13.5 & 17.6 \\
\hline $\begin{array}{l}\text { Number of maps included in the most probable class in the } \\
\text { analysis of the internal structures by the ASURA scheme }\end{array}$ & 301 & 204 & 212 & 194 & 167 \\
\hline Oversampling ratio§ & 161.8 & 14.1 & 321.3 & 11.1 & 14.6 \\
\hline
\end{tabular}

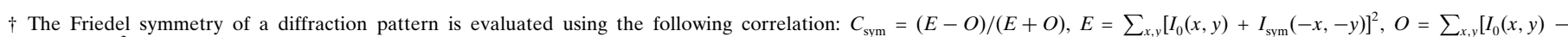

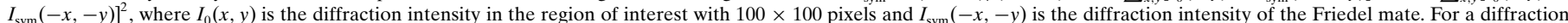

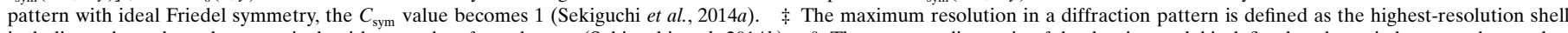

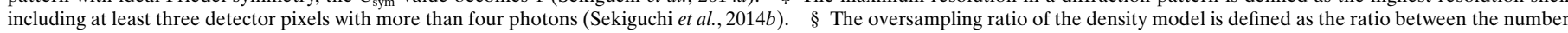

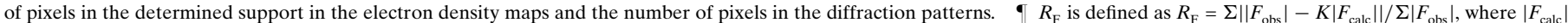

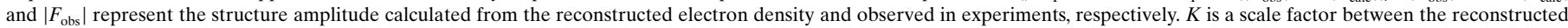

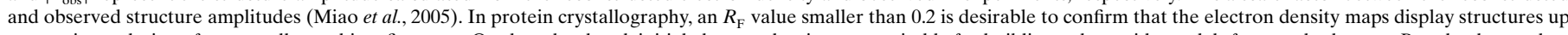

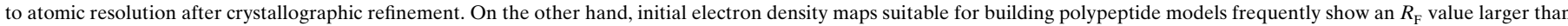

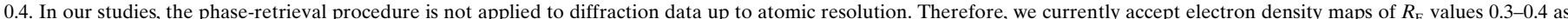

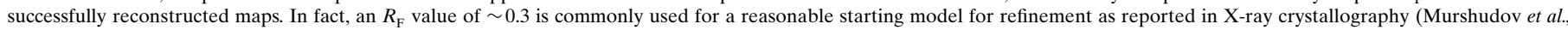

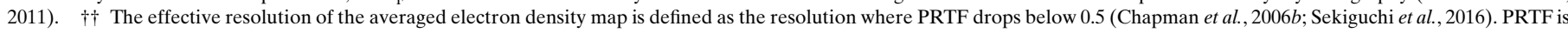
calculated from electron density maps of the most probable class selected in the multivariate analysis

direction. We currently control the amount of remaining buffer solution through inspection with optical microscope. However, semi-dried and heavily wet specimens appear depending on personal habits and skills in blotting. For specimen preparation without failures regarding the amount of buffer solution remaining around the specimen particles (Fig. 8), any robotics are particularly necessary to automatically control the blotting procedure with wick papers of regularized shapes and subsequent flash-freezing in liquid ethane. In addition, on the specimen disks with the arrayed PLL spots, an excess amount of specimen suspension can be removed easily by blotting, because the membrane surfaces without PLL spots are hydrophobic.

In raster scanning randomly dispersed biological particles, the probability of encountering diffraction patterns suitable

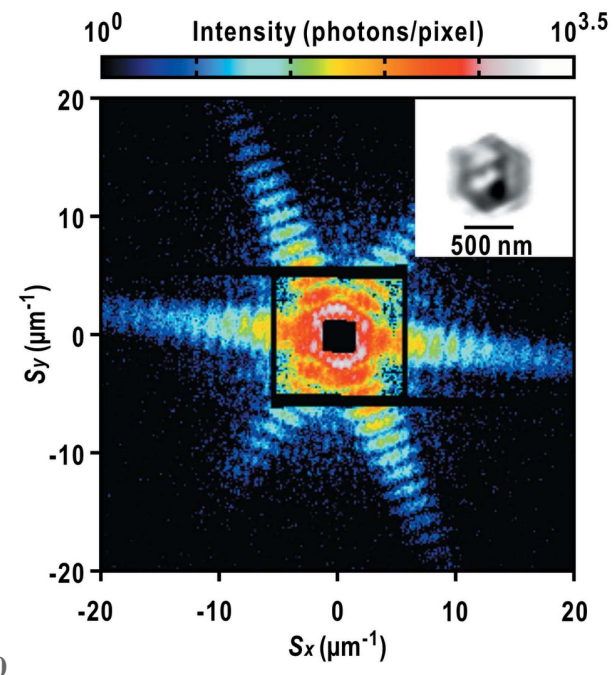

Figure 10

Diffraction pattern of a hexagonal ice crystal with sub-micrometer dimensions. The inset shows a retrieved electron density map projected along the direction of the incident X-rays. for structure analyses (Figs. $8 a, 9 a$ and $9 b$ ) is a few percent of all diffraction patterns. Therefore, an increase in the hit rate is necessary for diffraction data collection in a limited beam time. In this regard, successful data collection for specimen disks with regularly arrayed spots of PLL improves the efficiency of collecting diffraction patterns suitable for structure analyses.

The best method for stochastically collecting a large number of high-quality diffraction patterns is to increase events that $\mathrm{X}$-ray pulses hit specimen particles in a limited beam time. In our cryogenic experiments, the most time-consuming procedure is the exchange of the specimen holders. In this regard, the large specimen disks with multiple windows are advantageous for reducing the times of specimen exchanges. For instance, by using the TAKASAGO-6 diffraction apparatus, we can collect approximately 12000 diffraction patterns from one large silicon disk with nine SiN windows within $15 \mathrm{~min}$.

High-quality diffraction data are obtained when the centers of a particle and the focal spot of an XFEL pulse almost coincide. If specimen particles are ideally arrayed in the same pitch of raster scans and their positions are adjusted against focused XFEL pulses, almost all XFEL pulses would hit single particles. At SACLA, the positional fluctuation of the focused $\mathrm{X}$-ray beam is as small as $0.4 \mu \mathrm{m}$ at least for two days as we have reported (Oroguchi et al., 2015). Owing to the positional stability, better coincidence between particles and incident XFEL pulses would be possible by reducing the spot size of PLL to less than $2 \mu \mathrm{m}$, and controlling more accurately the distances between the PLL spots.

Through the present study, we conclude that a mechanically controlled blotting technique for the large disks, which are decorated by precisely arrayed PLL spots of diameter less than $2 \mu \mathrm{m}$, would be advantageous for obtaining high-quality diffraction patterns of single specimen particles through the collection of millions of diffraction patterns. 
4.2. Little thermal shrinkage and adiabatic expansion of cells during flash-cooling

Water occupies $60-70 \%$ of the volume of cells. When cells are set in a vacuum at ambient temperature, it is difficult to deny the probability that cellular structures are destroyed through the evaporation and bubbling of water inside the cells. In contrast, specimens embedded in vitreous ice are kept in the frozen-hydrated state in a vacuum of approximately $10^{-4} \mathrm{~Pa}$. The thermal shrinkage of the cytosols inside cells during flash-cooling would be as small as that of vitreous ice frozen from water. Cryogenic X-ray protein crystallography has shown that the linear thermal-expansion coefficient of a protein is $25 \times 10^{6} \mathrm{~K}^{-1}$ (Nakasako, 1999). Therefore, the thermal shrinkage of cellular specimens is negligibly small. In this study, this point is confirmed by the fact that the sizes of frozen-hydrated cyanobacterial cells are consistent with those of cyanobacteria in solution measured by DLS at ambient temperature (Fig. 9d).

In near-future experiments, frozen-hydrated biological cells or organelles harvested in a desired cell cycle would be subjected to two types of CXDI experiments. The first type is XFEL-CXDI experiments to obtain projection electron density maps of millions of cells using the TAKASAGO-6 apparatus equipped with a fast scan stage. The second type is cryogenic CXDI tomography experiments for single cells at synchrotron facilities (Rodriguez et al., 2015) using the KOTOBUKI-1 apparatus equipped with a high-precision goniometer within the limitation of radiation damage (Howells et al., 2009). Then, the two-dimensional electron density maps from the XFEL-CXDI experiments would be compared with the three-dimensional electron density maps from the CXDI tomography experiments. When biological cells have similar internal structures, this experimental scheme conducted for frozen-hydrated specimens would be helpful for visualizing the three-dimensional structures of cells. Therefore, cryogenic XFEL-CXDI experiments would contribute to visualize the structures inside cells and organelles dynamically varying in cell cycles.

\subsection{Contrast in projection electron density maps}

In the projection electron density maps retrieved from diffraction patterns of specimen particles in vitreous ice (Fig. 9), the fine structures inside the cells are clearly visualized rather than their outer shapes. In contrast, the overall shapes are clear as seen in the projection electron density maps retrieved from diffraction patterns of the cyanobacteria under semi-dried conditions (Fig. 8b) and in vacuum (van der Schot et al., 2015).

The substantial differences between the retrieved electron density maps of cells in vacuum and in solvent can be explained by the contrast variation theory in small-angle X-ray scattering (Fig. 11) (Ibel \& Stuhrmann, 1975). The excess electron density $\rho_{\mathrm{EX}}(\mathbf{r})$ of a particle from the uniform electron density of solvent $\rho_{\text {sol }}$ is expressed as

$$
\rho_{\mathrm{EX}}(\mathbf{r})=\Delta \rho \rho_{\mathrm{C}}(\mathbf{r})+\rho_{\mathrm{F}}(\mathbf{r}), \quad \Delta \rho=\bar{\rho}-\rho_{\mathrm{sol}},
$$
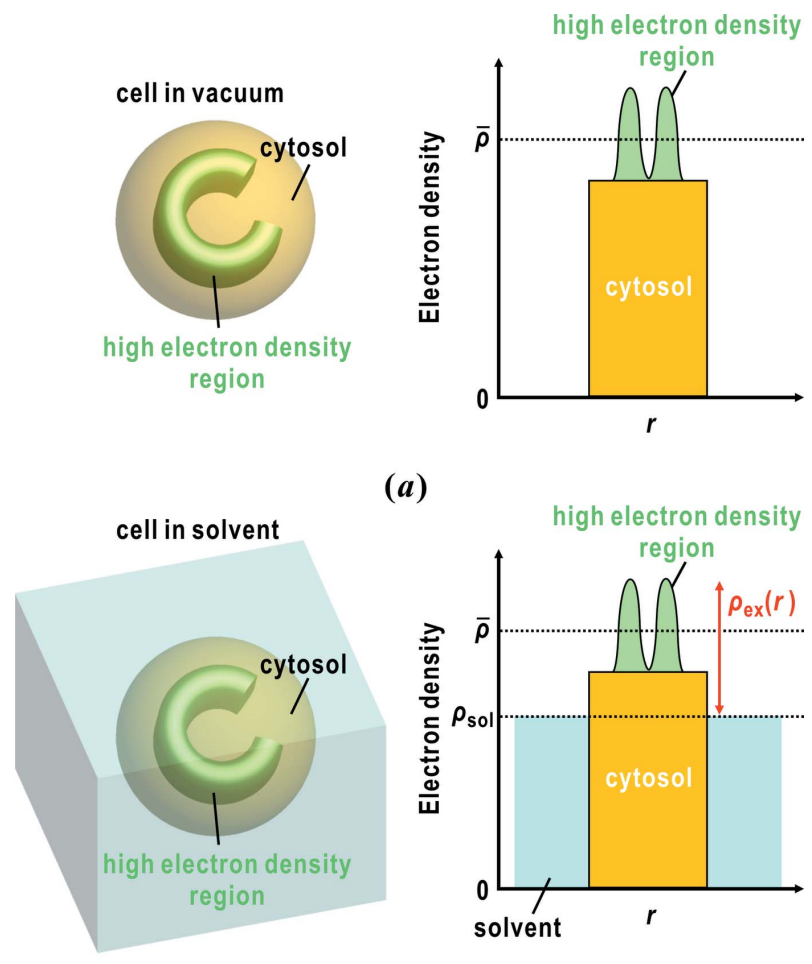

(a)

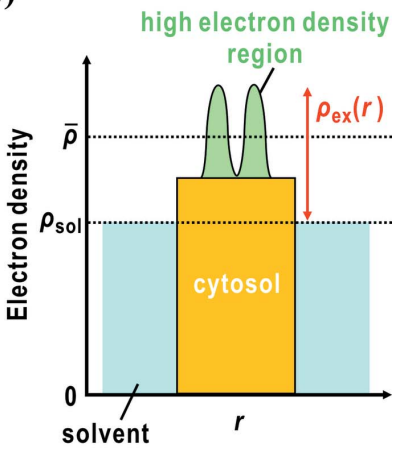

(b)

Figure 11

Schematic illustrations regarding the electron density contrast of biological cells in vacuum $(a)$ and in solvent $(b)$. The left panel shows a model system. The distribution of electron density in the model system is shown in the right panel.

where $\rho_{\mathrm{C}}(\mathbf{r})$ expresses the shape of the particle with average electron density $\bar{\rho} \cdot \rho_{\mathrm{C}}(\mathbf{r})$ takes a value of 1 inside the particle and 0 outside the particle. $\rho_{\mathrm{F}}(\mathbf{r})$ describes the spatial fluctuation of electron density from the average $\bar{\rho}$. X-rays diffracted by solvent with $\rho_{\text {sol }}$ within the beam size of incident X-rays are hidden by a beam stop because of the reciprocity between the size and the scattering vector. Then, the diffraction intensity at a scattering vector $(\mathbf{S}), I(\mathbf{S})$, from $\rho_{\mathrm{EX}}(\mathbf{r})$ is expressed as

$$
\begin{aligned}
I(\mathbf{S}) \propto & \Delta \rho^{2}\left|F_{\mathrm{C}}(\mathbf{S})\right|^{2}+\Delta \rho F_{\mathrm{C}}^{*}(\mathbf{S}) F_{\mathrm{F}}(\mathbf{S}) \\
& +\Delta \rho F_{\mathrm{F}}^{*}(\mathbf{r}) F_{\mathrm{C}}(\mathbf{S})+\left|F_{\mathrm{F}}(\mathbf{S})\right|^{2},
\end{aligned}
$$

where $F_{\mathrm{C}}(\mathbf{S})$ and $F_{\mathrm{F}}(\mathbf{S})$ are the structure factors of $\rho_{\mathrm{C}}(\mathbf{r})$ and $\rho_{\mathrm{F}}(\mathbf{r})$, respectively. Because $\rho_{\text {sol }}$ is almost zero for particles in vacuum, the diffraction intensity is dominated by the form factor from the overall shapes of specimen particles. In contrast, for specimen particles in vitreous ice or solvent, the diffraction intensity originating from the shape is reduced by the low contrast between $\bar{\rho}$ and $\rho_{\text {sol }}$. For instance, the $\bar{\rho}$ values of nucleic acids, proteins and water are $0.55,0.42$ and 0.33 electrons $\AA^{-3}$, respectively (Stuhrmann \& Miller, 1978; Svergun et al., 2013).

Therefore, biological particles in solvent or vitreous ice may be advantageous for extracting internal structures in the projection electron density maps. In the case of cyanobacteria, owing to the low contrast between the cells and the 
surrounding solvent, the structures inside cells, such as Cshaped high-electron-density regions, would appear clearly.

\subsection{Application of the preparation method to biological specimens}

The specimen preparation procedure here has been applied to various biological specimens. Recently, we obtained projection electron density maps of yeast nuclei, magnetic bacteria and chloroplasts of algae (Oroguchi et al., 2015; Takayama et al., 2015), whose sizes are smaller than the focal spot size of $2 \mu \mathrm{m}$ (Nakasako et al., 2013; Kobayashi et al., 2016). Specimens with small total scattering cross section give diffraction patterns with poor signal-to-noise ratio. Therefore, biological specimens of approximately $1 \mu \mathrm{m}$ size are desirable for XFEL-CXDI experiments at SACLA, when taking into account both the incident X-ray intensity and the focal spot size.

The number density of biological particles dispersed on PLL-coated membranes depends on the electrostatic interactions between the particles and the amino group of PLL. Cyanobacterial cells show good affinity to PLL. However, the affinity depends on, for instance, the amount of membrane proteins and other biological molecules existing on cell surfaces. Therefore, reagents with different types of functional groups would be necessary in some cases.

\section{Acknowledgements}

We thank Drs Kensuke Tono, Yuichi Inubushi and Takashi Kameshima of SACLA, and also Mr Tetsukon Kim, Takahiro Date, Toshiyuki Murakami and other members of the SACLA Engineering Team for their help in the alignment of the beamline optics and our diffraction apparatus. We also thank Dr Yasumasa Joti for his advice regarding the computation on the Mini-K supercomputer. The development of the diffraction apparatus was supported by grants for XFEL key technology and X-ray Free Electron Laser Priority Strategy Program from the MEXT to MN and MY. Specimen preparation and software development were supported by grants from the Japan Society for the Promotion of Science to MN (Nos. 1920402, 22018027 and 2465414), to YS (No. 15J01707) and to AK (No. 15J01831), from the Ministry of Education, Culture, Sports, Science and Technology of Japan to MN (Nos. 15076210, 20050030, 22244054) and to TO (No. 24113723). The XFEL-CXDI diffraction data were collected at BL3 of SACLA (proposal Nos. 2013A8043, 2013B8049, 2014A8033, 2014B8052 and 2015A8051). A part of the phase-retrieval calculations were carried out using the Mini-K supercomputer system at the SACLA facility (proposal Nos. 2014B8052 and 2015A8051).

\section{References}

Chapman, H. N. et al. (2006a). Nat. Phys. 2, 839-843.

Chapman, H. N., Barty, A., Marchesini, S., Noy, A., Hau-Riege, S. P.,

Cui, C., Howells, M. R., Rosen, R., He, H., Spence, J. C. H.,
Weierstall, U., Beetz, T., Jacobsen, C. \& Shapiro, D. (2006b). J. Opt. Soc. Am. A, 23, 1179-1200.

Chen, C. (1986). Lancet, 1, 884-886.

Ekeberg, T. et al. (2015). Phys. Rev. Lett. 114, 098102.

Emma, P. et al. (2010). Nat. Photon. 4, 641-647.

Fienup, J. R. (1982). Appl. Opt. 21, 2758-2769.

Gallagher-Jones, M., Bessho, Y., Kim, S., Park, J., Kim, S., Nam, D., Kim, C., Kim, Y., Noh, D.Y., Miyashita, O., Tama, F., Joti, Y., Kameshima, T., Hatsui, T., Tono, K., Kohmura, Y., Yabashi, M., Hasnain, S. S., Ishikawa, T. \& Song, C. (2014). Nat. Commun. 5, 3798.

Murshudov, G. N., Skubak, P., Lebedev, A. A., Pannu, N. S., Steiner, R. A., Nicholls, R. A., Winn, M. D., Long, F. \& Vagin, A. A. (2011). Acta Cryst. D67, 355-367.

Gibson, L. F. \& Khoury, J. T. (1986). Lett. Appl. Microbiol. 3, 127-129.

Grassucci, R. A., Taylor, D. J. \& Frank, J. (2007). Nat. Protoc. 2, 32393246.

Gutt, C., Wochner, P., Fischer, B., Conrad, H., Castro-Colin, M., Lee, S., Lehmkühler, F., Steinke, I., Sprung, M., Roseker, W., Zhu, D., Lemke, H., Bogle, S., Fuoss, P. H., Stephenson, G. B., Cammarata, M., Fritz, D. M., Robert, A. \& Grübel, G. (2012). Phys. Rev. Lett. 108, 024801.

Hantke, M. F. et al. (2014). Nat. Photon. 8, 943-949.

Hirata, K. et al. (2014). Nat. Methods, 11, 734-736.

Howells, M. R., Beetz, T., Chapman, H. N., Cui, C., Holton, J. M., Jacobsen, C. J., Kirz, J., Lima, E., Marchesini, S., Miao, H., Sayre, D., Shapiro, D. A., Spence, J. C. E. \& Starodub, D. (2009). J. Electron Spectrosc. Relat. Phenom. 170, 4-12.

Ibel, K. \& Stuhrmann, H. B. (1975). J. Mol. Biol. 93, 255-265.

Ishikawa, T. et al. (2012). Nat. Photon. 6, 540-544.

Jiang, H., Song, C., Chen, C. C., Xu, R., Raines, K. S., Fahimian, B. P., Lu, C. H., Lee, T. K., Nakashima, A., Urano, J., Ishikawa, T., Tamanoi, F. \& Miao, J. (2010). Proc. Natl Acad. Sci. USA, 107, 11234-11239.

Joti, Y., Kameshima, T., Yamaga, M., Sugimoto, T., Okada, K., Abe, T., Furukawa, Y., Ohata, T., Tanaka, R., Hatsui, T. \& Yabashi, M. (2015). J. Synchrotron Rad. 22, 571-576.

Kameshima, T., Ono, S., Kudo, T., Ozaki, K., Kirihara, Y., Kobayashi, K., Inubushi, Y., Yabashi, M., Horigome, T., Holland, A., Holland, K., Burt, D., Murao, H. \& Hatsui, T. (2014). Rev. Sci. Instrum. 85, 033110.

Kassemeyer, S. (2014). PhD Thesis, Free University of Berlin, Berlin, Germany.

Kassemeyer, S. et al. (2012). Opt. Express, 20, 4149-4158.

Kimura, T., Joti, Y., Shibuya, A., Song, C., Kim, S., Tono, K., Yabashi, M., Tamakoshi, M., Moriya, T., Oshima, T., Ishikawa, T., Bessho, Y. \& Nishino, Y. (2014). Nat. Commun. 5, 3052.

Kobayashi, A., Sekiguchi, Y., Takayama, Y., Oroguchi, T. \& Nakasako, M. (2014). Opt. Express, 22, 27892-27909.

Kobayashi, A., Sekiguchi, Y., Takayama, Y., Oroguchi, T. Shirahama, K., Torizuka, Y., Manoda, M., Nakasako, M., Yamamoto, M. (2016). Rev. Sci. Instrum. 87, 053109.

Kodama, W. \& Nakasako, M. (2011). Phys. Rev. E, 84, 021902.

Kuo, C. H., Chen, C. H. \& Huang, M. H. (2007). Adv. Funct. Mater. 17, 3773-3780

Lehmkühler, F., Gutt, C., Fischer, B., Schroer, M. A., Sikorski, M., Song, S., Roseker, W., Glownia, J., Chollet, M., Nelson, S., Tono, K., Katayama, T., Yabashi, M., Ishikawa, T., Robert, A. \& Grübel, G. (2014). Sci. Rep. 4, 5234.

Marchesini, S., He, H., Chapman, H. N., Hau-Riege, S. P., Noy, A., Howells, M. R., Weierstall, U. \& Spence, J. C. H. (2003). Phys. Rev. $B, \mathbf{6 8}, 140101$.

McDowall, A. W., Chang, J.-J., Freeman, R., Lepault, J., Walter, C. A. \& Dubochet, J. (1983). J. Microsc. 131, 1-9.

Medeiros, C. M. O., Forell, F., Oliveira, A. T. D. \& Rodrigues, J. L. (2002). Theriogenology, 57, 327-344.

Miao, J., Charalambous, P., Kirz, J. \& Sayre, D. (1999). Nature (London), 400, 342-344. 
Miao, J., Hodgson, K. O., Ishikawa, T., Larabell, C. A., LeGros, M. A. \& Nishino, Y. (2003b). Proc. Natl Acad. Sci. USA, 100, 110-112.

Miao, J., Ishikawa, T., Anderson, E. H. \& Hodgson, K. O. (2003a). Phys. Rev. B, 67, 174104.

Miao, J., Ishikawa, T., Johnson, B., Anderson, E. H., Lai, B. \& Hodgson, K. O. (2002). Phys. Rev. Lett. 89, 088303.

Miao, J., Ishikawa, T., Robinson, I. K. \& Murnane, M. M. (2015). Science, 348, 530-535.

Miao, J., Nishino, Y., Kohmura, Y., Johnson, B., Song, C., Risbud, S. H. \& Ishikawa, T. (2005). Phys. Rev. Lett. 95, 085503.

Moore, L. R., Coe, A., Zinser, E. R., Saito, M. A., Sullivan, M. B., Lindell, D., Frois-Moniz, K., Waterbury, J. \& Chisholm, S. W. (2007). Limnol. Oceanogr. Methods, 5, 353-362.

Nakasako, M. (1995). Nihon Kessho Gakkaishi, 37, 266-270.

Nakasako, M. (1999). J. Mol. Biol. 289, 547-564.

Nakasako, M., Takayama, Y., Oroguchi, T., Sekiguchi, Y., Kobayashi, A., Shirahama, K., Yamamoto, M., Hikima, T., Yonekura, K., MakiYonekura, S., Kohmura, Y., Inubushi, Y., Takahashi, Y., Suzuki, A., Matsunaga, S., Inui, Y., Tono, K., Kameshima, T., Joti, Y. \& Hoshi, T. (2013). Rev. Sci. Instrum. 84, 093705.

Nishino, Y., Takahashi, Y., Imamoto, N., Ishikawa, T. \& Maeshima, K. (2009). Phys. Rev. Lett. 102, 08101.

Oliver, W. (2004). PhD thesis, Swiss Federal Institute of Technology, Zurich, Switzerland (doi: 10.3929/ethz-a-004718257).

Oroguchi, T. \& Nakasako, M. (2013). Phys. Rev. E, 87, 022712.

Oroguchi, T. et al. (2015). J. Phys. B, 48, 184003.

Robarts, A. W. \& Sleytr, U. B. (1985). Low Temperature Methods in Biological Electron Microscopy. Amsterdam: Elsevier.

Robinson, I. K., Vartanyants, I. A., Williams, G. J., Pfeifer, M. A. \& Pitney, J. A. (2001). Phys. Rev. Lett. 87, 195505.

Rodriguez, J. A., Xu, R., Chen, C.-C., Huang, Z., Jiang, H., Chen, A. L., Raines, K. S., Pryor Jr, A., Nam, D., Wiegart, L., Song, C., Madsen, A., Chushkin, Y., Zontone, F., Bradley, P. J. \& Miao, J. (2015). IUCrJ, 2, 575-583.
Rodriguez, J. A., Xu, R., Chen, C.-C., Zou, Y. \& Miao, J. (2013). J. Appl. Cryst. 46, 312-318.

Schot, G. van der et al. (2015). Nat. Commun. 6, 5704.

Schwartz, O. (1986). Int. J. Oral Maxillofac. Surg. 15, 30-32.

Seibert, M. M. et al. (2011). Nature (London), 470, 78-81.

Sekiguchi, Y., Oroguchi, T. \& Nakasako, M. (2016). J. Synchrotron Rad. 23, 312-323.

Sekiguchi, Y., Oroguchi, T., Takayama, Y. \& Nakasako, M. (2014a). J. Synchrotron Rad. 21, 600-612.

Sekiguchi, Y., Yamamoto, M., Oroguchi, T., Takayama, Y., Suzuki, S. \& Nakasako, M. (2014b). J. Synchrotron Rad. 21, 1378-1383.

Song, C., Jiang, H., Mancuso, A., Amirbekian, B., Peng, L., Sun, R., Shah, S. S., Zhou, Z. H., Ishikawa, T. \& Miao, J. (2008). Phys. Rev. Lett. 101, 158101.

Stuhrmann, H. B. \& Miller, A. (1978). J. Appl. Cryst. 11, 325-345.

Svergun, D. I., Koch, M. H. J., Timmins, P. A. \& May, R. P. (2013). Small Angle X-ray and Neutron Scattering from Solutions of Biological Macromolecules. Oxford Science Publications.

Takayama, Y., Inui, Y., Sekiguchi, Y., Kobayashi, A., Oroguchi, T., Yamamoto, M., Matsunaga, S. \& Nakasako, M. (2015). Plant Cell Physiol. 56, 1272-1286.

Takayama, Y. \& Nakasako, M. (2012). Rev. Sci. Instrum. 83, 054301.

Ting, C. S., Hsieh, C., Sundararaman, S., Mannella, C. \& Marko, M. (2007). J. Bacteriol. 189, 4485-4493.

Tono, K., Togashi, T., Inubushi, Y., Sato, T., Katayama, T., Ogawa, K., Ohashi, H., Kimura, H., Takahashi, S., Takeshita, K., Tomizawa, H., Goto, S., Ishikawa, T. \& Yabashi, M. (2013). New J. Phys. 15, 083035 .

Watanabe, H. \& Kunitake, T. (2007). Adv. Mater. 19, 909-912.

Yumoto, H., Mimura, H., Koyama, T., Matsuyama, S., Tono, K., Togashi, T., Inubushi, Y., Sato, T., Tanaka, T., Kimura, T., Yokoyama, H., Kim, J., Sano, Y., Hachisu, Y., Yabashi, M., Ohashi, H., Ohmori, H., Ishikawa, T. \& Yamauchi, K. (2013). Nat. Photon. 7, 43-47. 